Palladium-Catalyzed Functionalization of Indoles with 2-Acetoxymethyl Substituted

\title{
Electron-deficient Alkenes
}

Shengming Ma, * Shichao Yu, Zhihua Peng, and Hao Guo

State Key Laboratory of Organometallic Chemistry, Shanghai Institute of Organic

Chemistry, Chinese Academy of Sciences, 354 Fenglin Lu, Shanghai 200032, P. R. China

masm@mail.sioc.ac.cn

Supporting Information

Table of Contents

\begin{tabular}{cc}
\hline Analytical data for compounds 3 & S1 \\
\hline $\begin{array}{c}{ }^{1} \mathrm{H} \text { NMR and }{ }^{13} \text { C NMR spectra of those } \\
\text { compounds }\end{array}$ & $\mathrm{S} 17$ \\
\hline
\end{tabular}




\section{1. $\mathrm{Pd}_{2}(\mathrm{dba})_{3} \cdot \mathrm{CHCl}_{3} / \mathrm{bpy}$-Catalyzed coupling of indoles with 2-acetoxymethyl substituted}

electron-deficient alkenes.

(1) 3-(2'-methoxycarbonyl-2'-propenyl)indole (3aa).<smiles>C=C(Cc1c[nH]c2ccccc12)C(C)=O</smiles>

Typical Procedure: A dried reaction tube equipped with a magnetic stirring bar was charged with indole 1a (59 mg, $0.5 \mathrm{mmol}$ ), methyl 2-acetoxymethylacrylate (96 mg, $0.6 \mathrm{mmol}$ ), $\mathrm{Pd}_{2}(\mathrm{dba})_{3} \cdot \mathrm{CHCl}_{3}(12.9 \mathrm{mg}, 2.5 \mathrm{~mol} \%)$, bipyridine $(7.8 \mathrm{mg}, 10 \mathrm{~mol} \%)$, and toluene $(1 \mathrm{~mL})$. Then the reaction was heated at $80{ }^{\circ} \mathrm{C}$ with stirring for 12 hours as monitored by TLC. Filtration of the reaction mixture through a small pad of silica gel $\left(\mathrm{Et}_{2} \mathrm{O}\right)$, concentration, and purification of the dark oil by flash chromatography on silica gel (eluent: ethyl acetate: petroleum ether $=1: 5)$ afforded 3aa as a yellow solid $(90 \mathrm{mg}, 84 \%)$. m.p. $69-71^{\circ} \mathrm{C}($ Hexane $)$; ${ }^{1} \mathrm{H}$ NMR $\left(\mathrm{CDCl}_{3}, 300 \mathrm{MHz}\right): \delta 8.04$ (bs, $\left.1 \mathrm{H}\right), 7.53(\mathrm{~d}, J=7.5 \mathrm{~Hz}, 1 \mathrm{H}), 7.36(\mathrm{~d}, J=8.1 \mathrm{~Hz}, 1$ H), $7.19(\mathrm{t}, J=8.4 \mathrm{~Hz}, 1 \mathrm{H}), 7.10(\mathrm{t}, J=8.1 \mathrm{~Hz}, 1 \mathrm{H}), 7.03(\mathrm{~d}, J=2.4 \mathrm{~Hz}, 1 \mathrm{H}), 6.20(\mathrm{~s}, 1 \mathrm{H})$, $5.49(\mathrm{~s}, 1 \mathrm{H}), 3.78(\mathrm{~s}, 2 \mathrm{H}), 3.76(\mathrm{~s}, 3 \mathrm{H}) ;{ }^{13} \mathrm{C} \mathrm{NMR}\left(\mathrm{CDCl}_{3}, 75.4 \mathrm{MHz}\right): \delta 167.8,139.3,136.2$, 127.2, 125.6, 122.8, 121.9, 119.2, 119.0, 112.6, 111.1, 51.9, 27.5 ppm; MS (70 eV): m/z (\%) $215\left(\mathrm{M}^{+}, 100\right)$; IR (neat): v 3378, 1697, 1628, 1433, 1424, 1310, 1287, 1141, $745 \mathrm{~cm}^{-1}$; HRMS calcd for $\mathrm{C}_{13} \mathrm{H}_{13} \mathrm{NO}_{2}$ 215.09462; Found: 215.09223. 
<smiles>C=C(Cc1c[nH]c2cccc(OCc3ccccc3)c12)C(C)=O</smiles>

The reaction of indole $\mathbf{1 b}(112 \mathrm{mg}, 0.5 \mathrm{mmol})$ with methyl 2-acetoxymethyl acrylate $\mathbf{2 a}$ (96 mg, $0.6 \mathrm{mmol})$ in the presence of $\mathrm{Pd}_{2}(\mathrm{dba})_{3} \cdot \mathrm{CHCl}_{3}(13 \mathrm{mg}, 2.5 \mathrm{~mol} \%)$ and bipyridine $(7.8$ $\mathrm{mg}, 10 \mathrm{~mol} \%)$ in $1 \mathrm{~mL}$ of toluene for $12 \mathrm{~h}$ afforded $121 \mathrm{mg}(76 \%)$ of 3ba: oil; ${ }^{1} \mathrm{H}$ NMR $\left(\mathrm{CDCl}_{3}, 300 \mathrm{MHz}\right): \delta 8.19(\mathrm{bs}, 1 \mathrm{H}), 7.60-7.32(\mathrm{~m}, 5 \mathrm{H}), 7.12(\mathrm{t}, J=7.8 \mathrm{~Hz}, 1 \mathrm{H}), 6.99(\mathrm{~d}, J=$ $8.1 \mathrm{~Hz}, 1 \mathrm{H}), 6.88(\mathrm{~s}, 1 \mathrm{H}), 6.59(\mathrm{~d}, J=7.5 \mathrm{~Hz}, 1 \mathrm{H}), 6.21(\mathrm{~s}, 1 \mathrm{H}), 5.37(\mathrm{~s}, 1 \mathrm{H}), 5.17(\mathrm{~s}, 2 \mathrm{H})$, 3.98 (s, $2 \mathrm{H}), 3.74$ (s, $3 \mathrm{H}) ;{ }^{13} \mathrm{C} \mathrm{NMR}\left(\mathrm{CDCl}_{3}, 75.4 \mathrm{MHz}\right): \delta 168.0,153.7,140.8,138.1,137.3$, $128.3,127.5,127.3,125.4,122.6,121.7,117.3,112.8,104.7,100.4,69.6,51.6,28.8$ ppm; MS $(70 \mathrm{eV}) \mathrm{m} / \mathrm{z}(\%) 230(100), 321\left(\mathrm{M}^{+}, 93.94\right) ; \mathrm{IR}$ (neat): $v 3408,1712,1631 \mathrm{~cm}^{-1} ; \mathrm{HRMS} \mathrm{m} / \mathrm{z}$ (MALDI) calcd for $\mathrm{C}_{20} \mathrm{H}_{20} \mathrm{NO}_{3}^{+}\left(\mathrm{M}^{+}+\mathrm{H}\right)$ 322.1438; Found: 322.1439 .

(3) 3-(2'-Methoxycarbonyl-2'-propenyl)-5-benzyloxyindole (3ca):<smiles>C=C(Cc1c[nH]c2ccc(OCc3ccccc3)cc12)C(C)=O</smiles>

The reaction of indole 1c $(112 \mathrm{mg}, 0.5 \mathrm{mmol})$ with methyl 2-acetoxymethyl acrylate 2a (96 mg, $0.6 \mathrm{mmol})$ in the presence of $\mathrm{Pd}_{2}(\mathrm{dba})_{3} \cdot \mathrm{CHCl}_{3}(13 \mathrm{mg}, 2.5 \mathrm{~mol} \%)$ and bipyridine (7.8 $\mathrm{mg}, 10 \mathrm{~mol} \%)$ in $1 \mathrm{~mL}$ of toluene for $12 \mathrm{~h}$ afforded $115 \mathrm{mg}(72 \%)$ of 3ca: oil; ${ }^{1} \mathrm{H}$ NMR $\left(\mathrm{CDCl}_{3}, 300 \mathrm{MHz}\right): \delta 7.98$ (bs, $\left.1 \mathrm{H}\right), 7.48$ (d, J = 7.8 Hz, $\left.2 \mathrm{H}\right), 7.40-7.19$ (m, $\left.3 \mathrm{H}\right), 7.27$ (d, $J$ $=7.8 \mathrm{~Hz}, 1 \mathrm{H}), 7.06-6.90(\mathrm{~m}, 3 \mathrm{H}), 6.18(\mathrm{~s}, 1 \mathrm{H}), 5.46(\mathrm{~s}, 1 \mathrm{H}), 5.08(\mathrm{~s}, 2 \mathrm{H}), 3.77(\mathrm{~s}, 3 \mathrm{H})$, 
3.73 (s, $2 \mathrm{H}) ;{ }^{13} \mathrm{C} \mathrm{NMR}\left(\mathrm{CDCl}_{3}, 75.4 \mathrm{MHz}\right): \delta 167.8,152.9,139.1,137.5,131.6,128.4,127.7$, $127.6,127.5,125.6,123.7,112.6,112.1,111.8,102.5,70.9,51.8,27.5 \mathrm{ppm} ; \mathrm{MS}(70 \mathrm{eV}) \mathrm{m} / \mathrm{z}$ (\%) $321\left(\mathrm{M}^{+}, 100\right)$; IR (neat): $v$ 3414, 1715, $1628 \mathrm{~cm}^{-1}$; HRMS m/z (MALDI) calcd for $\mathrm{C}_{20} \mathrm{H}_{20} \mathrm{NO}_{3}^{+}\left(\mathrm{M}^{+}+\mathrm{H}\right)$ 322.1438; Found: 322.1441 .

(4) 3-(2'-Methoxycarbonyl-2'-propenyl)-6-benzyloxyindole (3da):<smiles>C=C(Cc1c[nH]c2cc(OCc3ccccc3)ccc12)C(C)=O</smiles>

The reaction of indole 1d (112 $\mathrm{mg}, 0.5 \mathrm{mmol})$ with methyl 2-acetoxymethyl acrylate 2a (96 mg, $0.6 \mathrm{mmol})$ in the presence of $\mathrm{Pd}_{2}\left(\mathrm{dba}_{3} \cdot \mathrm{CHCl}_{3}(13 \mathrm{mg}, 2.5 \mathrm{~mol} \%)\right.$ and bipyridine ( 7.8 $\mathrm{mg}, 10 \mathrm{~mol} \%)$ in $1 \mathrm{~mL}$ of toluene for $12 \mathrm{~h}$ afforded $119 \mathrm{mg}$ (74\%) of 3da: oil; ${ }^{1} \mathrm{H}$ NMR ( $\left.\mathrm{CDCl}_{3}, 300 \mathrm{MHz}\right): \delta 7.98(\mathrm{bs}, 1 \mathrm{H}), 7.50-7.28(\mathrm{~m}, 6 \mathrm{H}), 6.95-6.82(\mathrm{~m}, 3 \mathrm{H}), 6.23(\mathrm{~s}, 1 \mathrm{H})$, $5.52(\mathrm{~s}, 1 \mathrm{H}), 5.09(\mathrm{~s}, 2 \mathrm{H}), 3.78(\mathrm{~s}, 3 \mathrm{H}), 3.77(\mathrm{~s}, 2 \mathrm{H}) ;{ }^{13} \mathrm{C} \mathrm{NMR}\left(\mathrm{CDCl}_{3}, 75.4 \mathrm{MHz}\right): \delta$ $167.9,155.5,139.4,137.3,136.9,128.5,127.8,127.4,125.5,121.9,121.7,119.6,112.6$, 110.0, 96.0, 70.5, 51.8, $27.6 \mathrm{ppm}$; MS (70 eV) m/z (\%) $230(100), 321\left(\mathrm{M}^{+}, 27.6\right) ; \mathrm{IR}$ (neat): $v$ $3411,1716,1629 \mathrm{~cm}^{-1}$; HRMS m/z (MALDI) calcd for $\mathrm{C}_{20} \mathrm{H}_{20} \mathrm{NO}_{3}{ }^{+}\left(\mathrm{M}^{+}+\mathrm{H}\right) 322.1438$; Found: 322.1440.

(5) 3-(2'-Methoxycarbonyl-2'-propenyl)-4-bromoindole (3ea): 
<smiles>C=C(Cc1c[nH]c2cccc(Br)c12)C(C)=O</smiles>

The reaction of indole 1 e $(98 \mathrm{mg}, 0.5 \mathrm{mmol})$ with methyl 2-acetoxymethyl acrylate (96 $\mathrm{mg}, 0.6 \mathrm{mmol})$ in the presence of $\mathrm{Pd}_{2}(\mathrm{dba})_{3} \cdot \mathrm{CHCl}_{3}(13 \mathrm{mg}, 2.5 \mathrm{~mol} \%)$ and bipyridine $(7.8 \mathrm{mg}$, $10 \mathrm{~mol} \%)$ in $1 \mathrm{~mL}$ of toluene for $10 \mathrm{~h}$ afforded $105 \mathrm{mg}(72 \%)$ of 3ea: solid, m.p. $110-111^{\circ} \mathrm{C}$; ${ }^{1} \mathrm{H}$ NMR $\left(\mathrm{CDCl}_{3}, 300 \mathrm{MHz}\right): \delta 8.23$ (bs, $\left.1 \mathrm{H}\right), 7.24-7.15$ (m, $\left.2 \mathrm{H}\right), 6.95-6.87$ (m, $\left.2 \mathrm{H}\right), 6.18$ (s, $1 \mathrm{H}), 5.24(\mathrm{~s}, 1 \mathrm{H}), 3.93(\mathrm{~s}, 2 \mathrm{H}), 3.71(\mathrm{~s}, 3 \mathrm{H}) ;{ }^{13} \mathrm{C} \mathrm{NMR}\left(\mathrm{CDCl}_{3}, 75.4 \mathrm{MHz}\right): \delta 167.9,140.8$, 137.6, 126.2, 125.4, 124.6, 124.0, 122.9, 114.3, 113.7, 110.5, 51.9, 28.1 ppm; MS (70 eV): m/z (\%): 43 (100), $293\left(\mathrm{M}^{+}\left({ }^{79} \mathrm{Br}\right), 61.56\right), 295\left(\mathrm{M}^{+}\left({ }^{81} \mathrm{Br}\right), 59.46\right)$; IR (neat): v 3363, 1705, 1631, 1436, 1142, $736 \mathrm{~cm}^{-1}$; HRMS calcd for $\mathrm{C}_{12} \mathrm{H}_{9} \mathrm{BrNO}_{2}\left(\mathrm{M}^{+}-\mathrm{CH}_{3}\right)$ 277.98167; Found: 277.98377.

(6) 3-(2'-Methoxycarbonyl-2'-propenyl)-5-bromoindole (3fa):<smiles>C=C(Cc1c[nH]c2ccc(Br)cc12)C(C)=O</smiles>

The reaction of indole $\mathbf{1 f}(98 \mathrm{mg}, 0.5 \mathrm{mmol})$ with methyl 2-acetoxymethyl acrylate $2 \mathbf{a}$ (96 $\mathrm{mg}, 0.6 \mathrm{mmol})$ in the presence of $\mathrm{Pd}_{2}(\mathrm{dba})_{3} \cdot \mathrm{CHCl}_{3}(13 \mathrm{mg}, 2.5 \mathrm{~mol} \%)$ and bipyridine $(7.8 \mathrm{mg}$, $10 \mathrm{~mol} \%)$ in $1 \mathrm{~mL}$ of toluene for $10 \mathrm{~h}$ afforded $101 \mathrm{mg}(69 \%)$ of $3 \mathbf{f a}$ : oil; ${ }^{1} \mathrm{H} \mathrm{NMR}\left(\mathrm{CDCl}_{3}\right.$, $300 \mathrm{MHz}): \delta 8.30(\mathrm{bs}, 1 \mathrm{H}), 7.66(\mathrm{~s}, 1 \mathrm{H}), 7.25(\mathrm{~d}, J=8.7 \mathrm{~Hz}, 1 \mathrm{H}), 7.18(\mathrm{~d}, J=8.7 \mathrm{~Hz}, 1 \mathrm{H})$, $6.99(\mathrm{~s}, 1 \mathrm{H}), 6.23(\mathrm{~s}, 1 \mathrm{H}), 5.50(\mathrm{~s}, 1 \mathrm{H}), 3.78(\mathrm{~s}, 3 \mathrm{H}), 3.73(\mathrm{~s}, 2 \mathrm{H}) ;{ }^{13} \mathrm{C} \mathrm{NMR}\left(\mathrm{CDCl}_{3}, 75.4\right.$ 
$\mathrm{MHz}): \delta 167.7,139.0,134.9,128.9,125.9,124.7,124.1,121.5,112.6,112.5,112.2,51.9$, 27.4 ppm; MS (70 eV) m/z (\%) 154 (100), $293\left(\mathrm{M}^{+}\left({ }^{79} \mathrm{Br}\right), 98.56\right), 295\left(\mathrm{M}^{+}\left({ }^{91} \mathrm{Br}\right), 95.05\right)$; IR (neat): $v$ 3418, 1707, $1631 \mathrm{~cm}^{-1}$; HRMS m/z (MALDI) calcd for $\mathrm{C}_{13} \mathrm{H}_{13} \mathrm{NO}_{2}{ }^{79} \mathrm{Br}^{+}\left(\mathrm{M}^{+}+\mathrm{H}\right)$ 294.0124; Found: 294.0133.

(7) 3-(2'-Methoxycarbonyl-2'-propenyl)-6-bromoindole (3ga):<smiles>C=C(Cc1c[nH]c2cc(Br)ccc12)C(C)=O</smiles>

The reaction of indole $\mathbf{1 g}$ (98 $\mathrm{mg}, 0.5 \mathrm{mmol}$ ) with methyl 2-acetoxymethyl acrylate (96 $\mathrm{mg}, 0.6 \mathrm{mmol})$ in the presence of $\mathrm{Pd}_{2}(\mathrm{dba})_{3} \cdot \mathrm{CHCl}_{3}(13 \mathrm{mg}, 2.5 \mathrm{~mol} \%)$ and bipyridine $(7.8 \mathrm{mg}$, $10 \mathrm{~mol} \%)$ in $1 \mathrm{~mL}$ of toluene for $10 \mathrm{~h}$ afforded $100 \mathrm{mg}(68 \%)$ of 3ga: oil; ${ }^{1} \mathrm{H} \mathrm{NMR}\left(\mathrm{CDCl}_{3}\right.$, $300 \mathrm{MHz}): \delta 8.23(\mathrm{bs}, 1 \mathrm{H}), 7.47$ (s, $1 \mathrm{H}), 7.39(\mathrm{~d}, J=8.4 \mathrm{~Hz}, 1 \mathrm{H}), 7.19(\mathrm{dd}, J=1.8,8.4 \mathrm{~Hz}$ $1 \mathrm{H}), 6.97(\mathrm{~s}, 1 \mathrm{H}), 6.22(\mathrm{~s}, 1 \mathrm{H}), 5.49(\mathrm{~s}, 1 \mathrm{H}), 3.77(\mathrm{~s}, 3 \mathrm{H}), 3.76(\mathrm{~s}, 2 \mathrm{H}) ;{ }^{13} \mathrm{C} \mathrm{NMR}\left(\mathrm{CDCl}_{3}\right.$, 75.4 MHz): $\delta 167.7,139.2,137.0,126.1,125.7,123.4,122.6,120.3,115.5,114.0,113.0,51.9$, 27.5 ppm; MS (70 eV) m/z (\%) 156 (100), $293\left(\mathrm{M}^{+}\left({ }^{79} \mathrm{Br}\right), 7.89\right), 295\left(\mathrm{M}^{+}\left({ }^{81} \mathrm{Br}\right), 7.43\right)$; IR (neat): $v$ 3420, 1709, 1630, 1454, $1144 \mathrm{~cm}^{-1}$; HRMS calcd for $\mathrm{C}_{12} \mathrm{H}_{9} \mathrm{BrNO}_{2}\left(\mathrm{M}^{+}-\mathrm{CH}_{3}\right)$ 277.98167; Found: 277.98203.

(8) 3-(2'-Methoxycarbonyl-2'-propenyl)-5-methylindole (3ha):<smiles>C=C(Cc1c[nH]c2ccc(C)cc12)C(C)=O</smiles> 
The reaction of indole $\mathbf{1 h}(66 \mathrm{mg}, 0.5 \mathrm{mmol})$ with methyl 2-acetoxymethyl acrylate (96 $\mathrm{mg}, 0.6 \mathrm{mmol})$ in the presence of $\mathrm{Pd}_{2}(\mathrm{dba})_{3} \cdot \mathrm{CHCl}_{3}(13 \mathrm{mg}, 2.5 \mathrm{~mol} \%)$ and bipyridine $(7.8 \mathrm{mg}$, $10 \mathrm{~mol} \%)$ in $1 \mathrm{~mL}$ of toluene for $12 \mathrm{~h}$ afforded $84 \mathrm{mg}(73 \%)$ of 3ha: oil; ${ }^{1} \mathrm{H} \mathrm{NMR}\left(\mathrm{CDCl}_{3}\right.$, $300 \mathrm{MHz}): \delta 7.96(\mathrm{bs}, 1 \mathrm{H}), 7.31(\mathrm{~s}, 1 \mathrm{H}), 7.25(\mathrm{~d}, J=8.1 \mathrm{~Hz}, 1 \mathrm{H}), 7.06-6.92(\mathrm{~m}, 2 \mathrm{H}), 6.20$ (s, $1 \mathrm{H}), 5.49$ (s, $1 \mathrm{H}), 3.78$ (s, $3 \mathrm{H}), 3.76(\mathrm{~s}, 2 \mathrm{H}), 2.46$ (s, $3 \mathrm{H}) ;{ }^{13} \mathrm{C} \mathrm{NMR}\left(\mathrm{CDCl}_{3}, 75.4 \mathrm{MHz}\right)$ : $\delta 167.9,139.4,134.6,128.6,127.5,125.6,123.6,123.0,118.7,112.2,110.8,51.9,27.5,21.5$ ppm; MS (70 eV): m/z (\%) 156 (100), 229 (M+1 19.69); IR (neat): v 3412, 1713, 1630, 1437, 1139, $794 \mathrm{~cm}^{-1}$; HRMS calcd for $\mathrm{C}_{14} \mathrm{H}_{15} \mathrm{NO}_{2}$ 229.11028; Found: 229.11186 .

\section{(9) 3-(2'-Methoxycarbonyl-2'-propenyl)-5-methoxyindole (3ia):}<smiles>C=C(Cc1c[nH]c2ccc(OC)cc12)C(C)=O</smiles>

The reaction of indole $1 \mathbf{i}(74 \mathrm{mg}, 0.5 \mathrm{mmol})$ with methyl 2-acetoxymethyl acrylate (96 $\mathrm{mg}, 0.6 \mathrm{mmol})$ in the presence of $\mathrm{Pd}_{2}(\mathrm{dba})_{3} \cdot \mathrm{CHCl}_{3}(13 \mathrm{mg}, 2.5 \mathrm{~mol} \%)$ and bipyridine $(7.8 \mathrm{mg}$, $10 \mathrm{~mol} \%)$ in $1 \mathrm{~mL}$ of toluene for $10 \mathrm{~h}$ afforded $93 \mathrm{mg}(76 \%)$ of 3ia: oil; ${ }^{1} \mathrm{H} \mathrm{NMR}\left(\mathrm{CDCl}_{3}\right.$, $300 \mathrm{MHz}): \delta 7.99$ (bs, $1 \mathrm{H}), 7.25$ (d, $J=8.4 \mathrm{~Hz}, 1 \mathrm{H}), 7.00(\mathrm{~d}, J=2.4 \mathrm{~Hz}, 1 \mathrm{H}), 6.95$ (d, $J=$ $2.1 \mathrm{~Hz}, 1 \mathrm{H}), 6.85$ (dd, J = 2.4, 8.4 Hz, $1 \mathrm{H}), 6.21(\mathrm{~s}, 1 \mathrm{H}), 5.48$ (s, $1 \mathrm{H}), 3.84$ (s, $3 \mathrm{H}), 3.78$ (s, $3 \mathrm{H}), 3.75(\mathrm{~s}, 2 \mathrm{H}) ;{ }^{13} \mathrm{C} \mathrm{NMR}\left(\mathrm{CDCl}_{3}, 75.4 \mathrm{MHz}\right): \delta 167.9,153.9,139.3,131.4,127.7,125.6$, 123.6, 112.4, 112.1, 111.8, 100.8, 55.9, 51.9, 27.6 ppm; MS (70 eV) m/z (\%) 59 (100), 245 $\left(\mathrm{M}^{+}, 33\right)$; IR (neat): $v 3411,1717,1629,1487,1140 \mathrm{~cm}^{-1}$; HRMS calcd for $\mathrm{C}_{14} \mathrm{H}_{15} \mathrm{NO}_{3}$ 245.10519; Found: 245.10475. 
(10) 3-(2'-Methoxycarbonyl-2'-propenyl)-2-methylindole (3ja):<smiles>C=C(COC)Cc1c(C)[nH]c2ccccc12</smiles>

The reaction of indole $\mathbf{1 j}$ ( $66 \mathrm{mg}, 0.5 \mathrm{mmol}$ ) with methyl 2-acetoxymethyl acrylate $\mathbf{2 a}$ (96 $\mathrm{mg}, 0.6 \mathrm{mmol})$ in the presence of $\mathrm{Pd}(\mathrm{dba})_{2}(14.4 \mathrm{mg}, 5 \mathrm{~mol} \%)$ and bipyridine $(7.8 \mathrm{mg}, 10$ mol\%) in $1 \mathrm{~mL}$ of toluene for $23 \mathrm{~h}$ afforded $77 \mathrm{mg}(67 \%)$ of 3ja: oil; ${ }^{1} \mathrm{H}$ NMR $\left(\mathrm{CDCl}_{3}, 300\right.$ MHz): $\delta 7.96$ (bs, $1 \mathrm{H}), 7.45$ (d, J=7.5 Hz, $1 \mathrm{H}), 7.27$ (d, J=7.5 Hz, $1 \mathrm{H}), 7.18-7.05$ (m, 2 $\mathrm{H}), 6.20(\mathrm{~s}, 1 \mathrm{H}), 5.39-5.36(\mathrm{~m}, 1 \mathrm{H}), 3.81(\mathrm{~s}, 3 \mathrm{H}), 3.74(\mathrm{~s}, 2 \mathrm{H}), 2.35(\mathrm{~s}, 3 \mathrm{H}),{ }^{13} \mathrm{C} \mathrm{NMR}$ $\left(\mathrm{CDCl}_{3}, 75.4 \mathrm{MHz}\right): \delta 167.9,138.9,135.2,132.3,128.5,125.2,120.8,119.1,118.1,110.2$, 107.5, 51.8, 26.3, 11.5 ppm; MS (70 eV) m/z (\%) 144 (100), $229\left(M^{+}, 58.24\right)$; IR (neat): $v$ 3400, 1720, $1630 \mathrm{~cm}^{-1}$; HRMS m/z (MALDI) calcd for $\mathrm{C}_{14} \mathrm{H}_{15} \mathrm{NO}_{2} \mathrm{Na}^{+}\left(\mathrm{M}^{+}+\mathrm{Na}\right) 252.0995$; Found: 252.1016.

(11) 3-(2'-Phenylsulfonyl-2'-propenyl)-1H-indole (3ac):<smiles>C=C(Cc1c[nH]c2ccccc12)S(=O)(=O)c1ccccc1</smiles>

The reaction of indole 1a $(59 \mathrm{mg}, 0.5 \mathrm{mmol})$ with 2-phenylsulfonyl-2-propenyl acetate 2c (144 mg, $0.6 \mathrm{mmol})$ in the presence of $\mathrm{Pd}_{2}(\mathrm{dba})_{3} \cdot \mathrm{CHCl}_{3}(12.9 \mathrm{mg}, 2.5 \mathrm{~mol} \%)$ and bipyridine (7.8 mg, $10 \mathrm{~mol} \%$ ) in $1 \mathrm{~mL}$ of toluene for $15 \mathrm{~h}$ afforded $111 \mathrm{mg}$ (75\%) of 3ac: white solid, m.p. $146-147^{\circ} \mathrm{C}$ (ethyl acetate/hexane); ${ }^{1} \mathrm{H}$ NMR $\left(\mathrm{CDCl}_{3}, 300 \mathrm{MHz}\right): \delta 8.02$ (bs, $\left.1 \mathrm{H}\right)$, 7.95-7.90 (m, 2 H), 7.67-7.62 (m, $1 \mathrm{H}), 7.58-7.51(\mathrm{~m}, 2 \mathrm{H}), 7.32(\mathrm{~d}, J=8.4 \mathrm{~Hz}, 1 \mathrm{H}), 7.16$ (t, 
$J=8.4 \mathrm{~Hz}, 1 \mathrm{H}), 7.09-6.95(\mathrm{~m}, 3 \mathrm{H}), 6.39(\mathrm{~s}, 1 \mathrm{H}), 5.55-5.51(\mathrm{~m}, 1 \mathrm{H}), 3.72(\mathrm{~s}, 2 \mathrm{H}) ;{ }^{13} \mathrm{C}$ NMR (DMSO, 75.4 MHz): $\delta$ 149.7, 138.5, 136.3, 133.8, 129.5, 127.8, 126.2, 124.8, 124.6, 121.1, 118.5, 117.7, 111.5, 108.2, 25.2 ppm; MS (70 eV) m/z (\%) 155 (100), $297\left(\mathrm{M}^{+}, 11.54\right)$; IR (neat): $v$ 3332, $1747 \mathrm{~cm}^{-1}$; HRMS $\mathrm{m} / \mathrm{z}$ (MALDI) calcd for $\mathrm{C}_{17} \mathrm{H}_{15} \mathrm{NO}_{2} \mathrm{SNa}^{+}\left(\mathrm{M}^{+}+\mathrm{Na}\right)$ 320.0716; Found: 320.0735 .

2. $\mathbf{P d}(\mathrm{acac})_{2} / \mathbf{P P h}_{3}$-Catalyzed coupling of $\mathrm{N}$-protected indoles with 2-acetoxymethyl substituted electron-deficient alkenes.

(1) 3-(2'-methoxycarbonyl-2'-propenyl)-1-methylindole (3ka):<smiles>C=C(Cc1cn(C)c2ccccc12)C(C)=O</smiles>

Typical Procedure: A dried reaction tube equipped with a magnetic stirring bar was charged with indole 1k (131 mg, $1.0 \mathrm{mmol})$, methyl 2-acetoxymethyl acrylate $2 \mathbf{a}(160 \mathrm{mg}, 1.0 \mathrm{mmol})$, $\mathrm{Pd}(\mathrm{acac})_{2}(15 \mathrm{mg}, 5 \mathrm{~mol} \%), \mathrm{PPh}_{3}(26 \mathrm{mg}, 10 \mathrm{~mol} \%)$, and HOAc $(0.5 \mathrm{~mL})$. Then the reaction was heated at $80{ }^{\circ} \mathrm{C}$ with stirring for 23 hours as monitored by TLC. Filtration of the reaction mixture through a small pad of silica gel $\left(\mathrm{Et}_{2} \mathrm{O}\right)$, concentration, and purification of the dark oil by flash chromatography on silica gel (eluent: ethyl acetate: petroleum ether $=1: 5$ ) afforded $192 \mathrm{mg}(84 \%)$ of 3ka: oil; ${ }^{1} \mathrm{H}$ NMR (300 MHz, $\left.\mathrm{CDCl}_{3}\right): \delta 7.51(\mathrm{~d}, J=7.8 \mathrm{~Hz}, 1 \mathrm{H}), 7.28$ (d, $J=7.8 \mathrm{~Hz}, 1 \mathrm{H}), 7.21(\mathrm{t}, J=7.8 \mathrm{~Hz}, 1 \mathrm{H}), 7.09(\mathrm{t}, J=7.8 \mathrm{~Hz}, 1 \mathrm{H}), 6.87(\mathrm{~s}, 1 \mathrm{H}), \quad 6.18(\mathrm{~s}, 1$ 
H), 5.51-5.46 (m, $1 \mathrm{H}), 3.75(\mathrm{~s}, 5 \mathrm{H}), 3.73(\mathrm{~s}, 3 \mathrm{H}) ;{ }^{13} \mathrm{C} \mathrm{NMR}\left(75 \mathrm{MHz}, \mathrm{CDCl}_{3}\right): \delta$ 167.6, $139.5,136.9,127.5,127.4,125.3,121.3,119.0,118.6,110.9,109.0,51.6,32.3,27.4$; IR(neat): 1720, $1631 \mathrm{~cm}^{-1} ;$ MS m/z (\%) $229\left(\mathrm{M}^{+}, 100\right)$; HRMS m/z (MALDI) calcd for $\mathrm{C}_{14} \mathrm{H}_{15} \mathrm{NO}_{2} \mathrm{Na}^{+}\left(\mathrm{M}^{+}+\mathrm{Na}\right)$ 252.0995; Found: 252.1000 .

(2) 3-(2'-Methoxycarbonyl-2'-propenyl)-1-buthylindole (3la)<smiles>C=C(Cc1cn(CCCC)c2ccccc12)C(=O)OC</smiles>

The reaction of 1-butylindole $\mathbf{1 l}(161 \mathrm{mg}, 1.0 \mathrm{mmol})$ with 2-acetoxymethyl acrylate 2a (192 $\mathrm{mg}, 1.2 \mathrm{mmol})$ in the presence of $\mathrm{Pd}(\mathrm{acac})_{2}(15 \mathrm{mg}, 5 \mathrm{~mol} \%)$ and $\mathrm{PPh}_{3}(26 \mathrm{mg}, 10 \mathrm{~mol} \%)$ in $2 \mathrm{~mL}$ of HOAc for $9 \mathrm{~h}$ afforded $219 \mathrm{mg}(81 \%)$ of 3la: yellow oil; ${ }^{1} \mathrm{H}$ NMR $(300 \mathrm{MHz}$, $\left.\mathrm{CDCl}_{3}\right): \delta 7.52(\mathrm{~d}, J=7.8 \mathrm{~Hz}, 1 \mathrm{H}), 7.32(\mathrm{~d}, J=7.5 \mathrm{~Hz}, 1 \mathrm{H}), 7.20(\mathrm{t}, J=6.9 \mathrm{~Hz}, 1 \mathrm{H}), 7.08$ (t, $J=8.1 \mathrm{~Hz}, 1 \mathrm{H}), 6.94(\mathrm{~s}, 1 \mathrm{H}), 6.19(\mathrm{~s}, 1 \mathrm{H}), 5.50-5.46(\mathrm{~m}, 1 \mathrm{H}), 4.08(\mathrm{t}, J=7.2 \mathrm{~Hz}, 2 \mathrm{H})$, $3.78(\mathrm{~s}, 3 \mathrm{H}), 3.77(\mathrm{~s}, 2 \mathrm{H}), 1.85-1.73(\mathrm{~m}, 2 \mathrm{H}), 1.40-1.25(\mathrm{~m}, 2 \mathrm{H}), 0.94(\mathrm{t}, J=7.2 \mathrm{~Hz}, 3 \mathrm{H})$;

${ }^{13} \mathrm{C} \mathrm{NMR}\left(75 \mathrm{MHz}, \mathrm{CDCl}_{3}\right): \delta 167.8,139.6,136.3,127.7,126.6,125.4,121.2,119.2,118.6$, 111.0, 109.3, 51.8, 45.8, 32.3, 27.5, 20.1, 13.7; IR(neat): 1721, $1631 \mathrm{~cm}^{-1}$; MS m/z (\%) 271 $\left(\mathrm{M}^{+}, 100\right)$; HRMS m/z (MALDI) calcd for $\mathrm{C}_{17} \mathrm{H}_{22} \mathrm{NO}_{2}{ }^{+}\left(\mathrm{M}^{+}+\mathrm{H}\right)$ 272.1645; Found: 272.1638 .

\section{(3) 3-(2'-Methoxycarbonyl-2'-propenyl)-1-allylindole (3ma)}




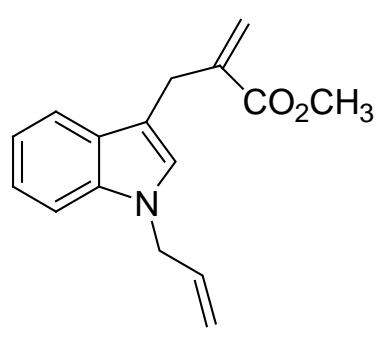

The reaction of 1-allylindole $\mathbf{1 m}(157 \mathrm{mg}, 1.0 \mathrm{mmol})$ with 2-acetoxymethyl acrylate $\mathbf{2 a}$ (192 mg, $1.2 \mathrm{mmol})$ in the presence of $\mathrm{Pd}(\mathrm{acac})_{2}(15 \mathrm{mg}, 5 \mathrm{~mol} \%)$ and $\mathrm{PPh}_{3}(26 \mathrm{mg}, 10 \mathrm{~mol} \%)$ in $2 \mathrm{~mL}$ of HOAc for $18 \mathrm{~h}$ afforded $189 \mathrm{mg}(74 \%)$ of 3ma: yellow oil; ${ }^{1} \mathrm{H}$ NMR (300 MHz, $\left.\mathrm{CDCl}_{3}\right): \delta 7.51(\mathrm{~d}, J=7.8 \mathrm{~Hz}, 1 \mathrm{H}), 7.29(\mathrm{~d}, J=7.8 \mathrm{~Hz}, 1 \mathrm{H}), 7.19(\mathrm{t}, J=7.8 \mathrm{~Hz}, 1 \mathrm{H}), 7.08$ (t, $J=7.8 \mathrm{~Hz}, 1 \mathrm{H}), 6.94(\mathrm{~s}, 1 \mathrm{H}), 6.19(\mathrm{~s}, 1 \mathrm{H}), 6.03-5.90(\mathrm{~m}, 1 \mathrm{H}), 5.51-5.48(\mathrm{~m}, 1 \mathrm{H}), 5.18$ $(\mathrm{d}, J=10.2 \mathrm{~Hz}, 1 \mathrm{H}), 5.05(\mathrm{~d}, J=17.7 \mathrm{~Hz}, 1 \mathrm{H}), 4.71-4.68(\mathrm{~m}, 2 \mathrm{H}), 3.77(\mathrm{~s}, 5 \mathrm{H}) ;{ }^{13} \mathrm{C} \mathrm{NMR}$ $\left(75 \mathrm{MHz}, \mathrm{CDCl}_{3}\right): \delta 167.8,139.5,136.4,133.5,127.8,126.5,125.5,121.5,119.2,118.9$, 117.1, 111.6, 109.6, 51.8, 48.6, 27.5; IR(neat): 1720, $1631 \mathrm{~cm}^{-1}$; MS m/z (\%) 170 (100), 255 $\left(\mathrm{M}^{+}, 99.57\right)$; HRMS $m / z$ (MALDI) calcd for $\mathrm{C}_{16} \mathrm{H}_{18} \mathrm{NO}_{2}{ }^{+}\left(\mathrm{M}^{+}+\mathrm{H}\right)$ 256.1332; Found: 256.1347.

(4) 3-(2'-Methoxycarbonyl-2'-propenyl)-1-benzylindole (3na)<smiles>C=C(Cc1cn(Cc2ccccc2)c2ccccc12)C(C)=O</smiles>

The reaction of 1-benzylindole $1 \mathrm{n}(100 \mathrm{mg}, 0.5 \mathrm{mmol})$ with 2-acetoxymethyl acrylate 2a (96 mg, $0.6 \mathrm{mmol})$ in the presence of $\mathrm{Pd}(\mathrm{acac})_{2}(7.5 \mathrm{mg}, 5 \mathrm{~mol} \%)$ and $\mathrm{PPh}_{3}(13 \mathrm{mg}, 10 \mathrm{~mol} \%)$ in $1 \mathrm{~mL}$ of HOAc for $15 \mathrm{~h}$ afforded $125 \mathrm{mg}$ (82\%) of 3na: yellow oil; ${ }^{1} \mathrm{H}$ NMR (300 MHz, $\left.\mathrm{CDCl}_{3}\right): \delta 7.61(\mathrm{~d}, J=7.5 \mathrm{~Hz}, 1 \mathrm{H}), 7.38-7.10(\mathrm{~m}, 8 \mathrm{H}), 7.02(\mathrm{~s}, 1 \mathrm{H}), 6.26(\mathrm{~s}, 1 \mathrm{H}), 5.56(\mathrm{~s}, 1$ H), $5.31(\mathrm{~s}, 2 \mathrm{H}), 3.85(\mathrm{~s}, 2 \mathrm{H}), 3.81(\mathrm{~s}, 3 \mathrm{H}),{ }^{13} \mathrm{C} \mathrm{NMR}\left(75 \mathrm{MHz}, \mathrm{CDCl}_{3}\right): \delta 167.7,139.5$, $137.6,136.7,128.6,127.9,127.4,126.9,126.6,125.5,121.7,119.2,119.0,111.9,109.7,51.8$, 
49.8, 27.5; IR(neat): 1719, $1630 \mathrm{~cm}^{-1}$; MS m/z (\%) 91 (100), $305\left(\mathrm{M}^{+}, 25.63\right)$; HRMS m/z (MALDI) calcd for $\mathrm{C}_{20} \mathrm{H}_{19} \mathrm{NO}_{2} \mathrm{Na}^{+}\left(\mathrm{M}^{+}+\mathrm{Na}\right)$ 328.1308; Found: 328.1316 .

(5) 3-(2'-methoxycarbonyl-2'-propenyl)-1,2-dimethylindole (3oa)<smiles>C=C(COC)Cc1c(C)n(C)c2ccccc12</smiles>

The reaction of 1,2-dimethylindole 10 (73 $\mathrm{mg}, 0.5 \mathrm{mmol})$ with 2-acetoxymethyl acrylate 2a (96 mg, $0.6 \mathrm{mmol})$ in the presence of $\mathrm{Pd}(\mathrm{acac})_{2}(7.5 \mathrm{mg}, 5 \mathrm{~mol} \%)$ and bipyridine $(13 \mathrm{mg}$, $10 \mathrm{~mol} \%$ ) in $1 \mathrm{~mL}$ of HOAc for $10 \mathrm{~h}$ afforded $87 \mathrm{mg}(72 \%)$ of 3oa: yellow oil; ${ }^{1} \mathrm{H}$ NMR (300 $\left.\mathrm{MHz}, \mathrm{CDCl}_{3}\right): \delta 7.50(\mathrm{~d}, J=7.8 \mathrm{~Hz}, 1 \mathrm{H}), 7.31(\mathrm{~d}, J=8.1 \mathrm{~Hz}, 1 \mathrm{H}), 7.21(\mathrm{t}, J=7.8 \mathrm{~Hz}, 1 \mathrm{H})$, $7.12(\mathrm{t}, J=7.8 \mathrm{~Hz}, 1 \mathrm{H}), 6.25-6.20(\mathrm{~m}, 1 \mathrm{H}), 5.42-5.38(\mathrm{~m}, 1 \mathrm{H}), 3.84(\mathrm{~s}, 3 \mathrm{H}), 3.80(\mathrm{~s}, 2 \mathrm{H})$, $3.69(\mathrm{~s}, 3 \mathrm{H}), 2.39(\mathrm{~s}, 3 \mathrm{H}) ;{ }^{13} \mathrm{C} \mathrm{NMR}\left(75 \mathrm{MHz}, \mathrm{CDCl}_{3}\right): \delta 167.8,139.1,136.5,134.1,127.6$, 125.1, 120.4, 118.7, 118.1, 108.4, 106.8, 51.7, 29.4, 26.5, 10.1; IR(neat): 1720, $1630 \mathrm{~cm}^{-1}$; MS m/z (\%) $158 \quad(100), 243 \quad\left(\mathrm{M}^{+}, 49.93\right) ; \quad$ HRMS $\mathrm{m} / \mathrm{z} \quad$ (MALDI) calcd for $\mathrm{C}_{15} \mathrm{H}_{17} \mathrm{NO}_{2} \mathrm{Na}^{+}\left(\mathrm{M}^{+}+\mathrm{Na}\right)$ 266.1152; Found: 266.1158 .

(6) 6-benzyloxy 3-(2'-methoxycarbonyl-2'-propenyl)-1-methylindole (3pa)<smiles>C=C(Cc1cn(C)c2cc(OCc3ccccc3)ccc12)C(C)=O</smiles>

The reaction of 6-benyloxy-1-allylindole 1p (119 mg, $0.5 \mathrm{mmol})$ with 2-acetoxymethyl 
acrylate $2 \mathbf{a}(96 \mathrm{mg}, 0.6 \mathrm{mmol})$ in the presence of $\mathrm{Pd}(\mathrm{acac})_{2}(7.5 \mathrm{mg}, 5 \mathrm{~mol} \%)$ and $\mathrm{PPh}_{3}(13$ $\mathrm{mg}, 10 \mathrm{~mol} \%$ ) in $1 \mathrm{~mL}$ of HOAc for $11 \mathrm{~h}$ afforded $125 \mathrm{mg}(75 \%)$ of 3pa: yellow oil; ${ }^{1} \mathrm{H}$ NMR $\left(300 \mathrm{MHz}, \mathrm{CDCl}_{3}\right): \delta 7.53(\mathrm{~d}, J=7.2 \mathrm{~Hz}, 2 \mathrm{H}), 7.48-7.32(\mathrm{~m}, 4 \mathrm{H}), 6.93-6.89(\mathrm{~m}, 2 \mathrm{H}), 6.82$ (s, $1 \mathrm{H}), 6.25(\mathrm{~s}, 1 \mathrm{H}), 5.57(\mathrm{~s}, 1 \mathrm{H}), 5.17(\mathrm{~s}, 2 \mathrm{H}), 3.81(\mathrm{~s}, 3 \mathrm{H}), 3.78(\mathrm{~s}, 2 \mathrm{H}), 3.69$ (s, $3 \mathrm{H})$;

${ }^{13} \mathrm{C}$ NMR $\left(75 \mathrm{MHz}, \mathrm{CDCl}_{3}\right): \delta 167.7,155.4,139.6,137.6,137.4,128.4,127.7,127.4,126.4$, 125.4, 122.3, 119.7, 111.2, 109.2, 94.2, 70.5, 51.7, 32.5, 27.5; IR(neat): 1719, $1625 \mathrm{~cm}^{-1}$; MS m/z (\%) 244 (100), $335\left(\mathrm{M}^{+}, 18.24\right)$; HRMS m/z (MALDI) calcd for $\mathrm{C}_{21} \mathrm{H}_{21} \mathrm{NO}_{3} \mathrm{Na}^{+}\left(\mathrm{M}^{+}+\mathrm{Na}\right)$ 358.1414; Found: 358.1419.

(7) 3-(2'-acetyl-2'-propenyl)-1-methylindole (3kb)<smiles>C=C(Cc1cn(C)c2ccccc12)C(C)=O</smiles>

The reaction of 1 -methylindole $\quad \mathbf{1 k} \quad(131 \quad \mathrm{mg}, \quad 1.0 \quad \mathrm{mmol})$ with 3-acetoxymethyl-3-buten-2-one $2 \mathbf{b}(170 \mathrm{mg}, 1.2 \mathrm{mmol})$ in the presence of $\mathrm{Pd}(\mathrm{acac})_{2}(15 \mathrm{mg}$, $5 \mathrm{~mol} \%$ ) and $\mathrm{PPh}_{3}(26 \mathrm{mg}, 10 \mathrm{~mol} \%)$ in $1 \mathrm{~mL}$ of HOAc for $22.5 \mathrm{~h}$ afforded $168 \mathrm{mg}(79 \%)$ of 3kb: oil; ${ }^{1} \mathrm{H}$ NMR (300 MHz, $\left.\mathrm{CDCl}_{3}\right): \delta 7.49(\mathrm{~d}, J=6.9 \mathrm{~Hz}, 1 \mathrm{H}), 7.29(\mathrm{~d}, J=7.8 \mathrm{~Hz}, 1 \mathrm{H})$, $7.21(\mathrm{t}, J=6.9 \mathrm{~Hz}, 1 \mathrm{H}), 7.08(\mathrm{t}, J=7.8 \mathrm{~Hz}, 1 \mathrm{H}), 6.87(\mathrm{~s}, 1 \mathrm{H}), 6.04(\mathrm{~s}, 1 \mathrm{H}), 5.70(\mathrm{~s}, 1 \mathrm{H})$, 3.74 (s, $3 \mathrm{H}), 3.72$ (s, $2 \mathrm{H}), 2.36$ (s, $3 \mathrm{H}) ;{ }^{13} \mathrm{C} \mathrm{NMR}\left(75 \mathrm{MHz}, \mathrm{CDCl}_{3}\right): \delta$ 199.6, 147.8, 136.9, $127.5,125.7,121.3,118.92,118.89,118.6,111.2,109.0,32.3,26.0,25.9$; IR(neat): 1675, $1626 \mathrm{~cm}^{-1} ; \mathrm{MS} \mathrm{m} / \mathrm{z}(\%) 213\left(\mathrm{M}^{+}, 100\right) ; \mathrm{HRMS} \mathrm{m} / \mathrm{z}$ (MALDI) calcd for $\mathrm{C}_{14} \mathrm{H}_{15} \mathrm{NONa}^{+}\left(\mathrm{M}^{+}+\mathrm{Na}\right)$ 236.1046; Found: 236.1052. 
(8) 3-(2-phenylsulfonyl-2-propenyl)-1-methylindole (3kc)

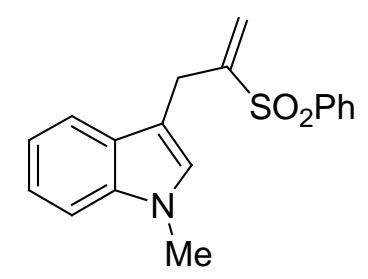

The reaction of 1-methylindole $1 \mathbf{k}(132 \mathrm{mg}, 1.0 \mathrm{mmol})$ with 2-phenylsulfonyl-2-propenyl acetate $2 \mathrm{c}(288 \mathrm{mg}, 1.2 \mathrm{mmol})$ in the presence of $\mathrm{Pd}(\mathrm{acac})_{2}(15 \mathrm{mg}, 5 \mathrm{~mol} \%)$ and $\mathrm{PPh}_{3}(26 \mathrm{mg}$, $10 \mathrm{~mol} \%$ ) in $2 \mathrm{~mL}$ of HOAc for $11 \mathrm{~h}$ afforded $236 \mathrm{mg}$ (76\%) of 3kc: white solid, m.p. $128-129^{\circ} \mathrm{C} ;{ }^{1} \mathrm{H}$ NMR $\left(300 \mathrm{MHz}, \mathrm{CDCl}_{3}\right): \delta 7.90(\mathrm{~d}, J=8.1 \mathrm{~Hz}, 2 \mathrm{H}), 7.62(\mathrm{t}, J=6.6 \mathrm{~Hz}, 1 \mathrm{H})$, $7.51(\mathrm{t}, J=7.8 \mathrm{~Hz}, 2 \mathrm{H}), 7.25(\mathrm{~d}, J=8.1 \mathrm{~Hz}, 1 \mathrm{H}), 7.20(\mathrm{t}, J=7.2 \mathrm{~Hz}, 1 \mathrm{H}), 7.10(\mathrm{~d}, J=7.8$ Hz, $1 \mathrm{H}), 7.00(\mathrm{t}, J=7.2 \mathrm{~Hz}, 1 \mathrm{H}), 6.79(\mathrm{~s}, 1 \mathrm{H}), 6.40(\mathrm{~s}, 1 \mathrm{H}), 5.57$ (s, $1 \mathrm{H}), 3.76(\mathrm{~s}, 2 \mathrm{H})$, $3.73(\mathrm{~s}, 3 \mathrm{H}) ;{ }^{13} \mathrm{C} \mathrm{NMR}\left(75 \mathrm{MHz}, \mathrm{CDCl}_{3}\right): \delta 149.7,138.9,136.9,133.3,129.0,128.09$, $128.08,128.05,127.0,124.7,121.7,119.0,118.6,109.2,108.2,32.6,25.6$; IR(neat): 1615, $1303 \mathrm{~cm}^{-1}$; MS m/z (\%) 169 (100), $311\left(\mathrm{M}^{+}, 8.46\right)$; HRMS m/z (MALDI) calcd for $\mathrm{C}_{18} \mathrm{H}_{18} \mathrm{NO}_{2} \mathrm{~S}^{+}\left(\mathrm{M}^{+}+\mathrm{H}\right)$ 312.1053; Found: 312.1044 .

(9) 3-(2'-cyano-2'-propenyl)-1-methylindole (3kd)

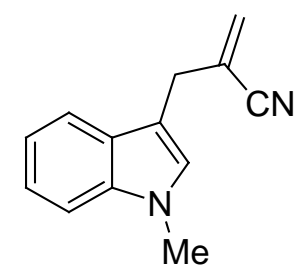

The reaction of 1-methylindole $\mathbf{1 k}(127 \mathrm{mg}, 1.0 \mathrm{mmol})$ with 2-acetoxymethyl acrylonitrile $2 \mathbf{d}(150 \mathrm{mg}, 1.2 \mathrm{mmol})$ in the presence of $\mathrm{Pd}(\mathrm{acac})_{2}(15 \mathrm{mg}, 5 \mathrm{~mol} \%)$ and $\mathrm{PPh}_{3}$ 
(25 mg, $10 \mathrm{~mol} \%$ ) in $2 \mathrm{~mL}$ of HOAc for $16 \mathrm{~h}$ afforded $143 \mathrm{mg}$ (75\%) of 3kd: white solid, m.p. 51-52 ${ }^{\circ} \mathrm{C}$ (ethyl acetate/Hexane); ${ }^{1} \mathrm{H}$ NMR $\left(300 \mathrm{MHz}, \mathrm{CDCl}_{3}\right): \delta 7.46(\mathrm{~d}, J=7.8 \mathrm{~Hz}, 1 \mathrm{H})$, 7.31-7.16 (m, $2 \mathrm{H}), 7.10(\mathrm{t}, J=8.1 \mathrm{~Hz}, 1 \mathrm{H}), 6.92(\mathrm{~s}, 1 \mathrm{H}), 5.84(\mathrm{~s}, 1 \mathrm{H}), 5.70(\mathrm{~s}, 1 \mathrm{H}), 3.70(\mathrm{~s}$, $3 \mathrm{H}), 3.65$ (s, $2 \mathrm{H}) ;{ }^{13} \mathrm{C}$ NMR $\left(75 \mathrm{MHz}, \mathrm{CDCl}_{3}\right): \delta 137.0,130.3,127.6,127.1,122.3,121.8$, $119.1,118.9,118.5,109.4,108.1,32.6,30.5 ;$ IR(neat): 2221, 1617, 1474, $742 \mathrm{~cm}^{-1}$; MS m/z (\%) 144 (100), $196\left(\mathrm{M}^{+}, 20.29\right)$; Anal. Calcd. for $\mathrm{C}_{13} \mathrm{H}_{12} \mathrm{~N}_{2}$ : (\%) C 79.56, H 6.16, N 14.27; Found: C 79.60, H 6.21, N 14.37.

(10) 3-(2'-methoxycarbonyl-1'-buten-3'-yl)-1-methylindole (3ke).<smiles>C=C(COC)C(C)c1cn(C)c2ccccc12</smiles>

The reaction of $1 \mathbf{k}(67 \mathrm{mg}, 0.5 \mathrm{mmol})$ with $2 \mathbf{e}(113 \mathrm{mg}, 0.6 \mathrm{mmol})$ in the presence of $\mathrm{Pd}(\mathrm{acac})_{2}(8 \mathrm{mg}, 5 \mathrm{~mol} \%)$ and $\mathrm{PPh}_{3}(13 \mathrm{mg}, 10 \mathrm{~mol} \%)$ in $2 \mathrm{~mL}$ of HOAc for $3 \mathrm{~h}$ afforded 80 $\mathrm{mg}(66 \%)$ of 3ke. Compound $2 \mathbf{e}(7 \mathrm{mg}, 6 \%)$ was recovered. The reaction of $\mathbf{1 k}(66 \mathrm{mg}, 0.5$ mmol) with $2 \mathrm{~g}(277 \mathrm{mg}, 1.5 \mathrm{mmol})$ in the presence of $\mathrm{Pd}(\mathrm{acac})_{2}(14 \mathrm{mg}, 10 \mathrm{~mol} \%)$ and $\mathrm{PPh}_{3}$ (26 mg, $20 \mathrm{~mol} \%$ ) in $2 \mathrm{~mL}$ of HOAc for $3.25 \mathrm{~h}$ afforded $62 \mathrm{mg}$ (51\%) of 3ke. Compound $\mathbf{2 g}$ (25 mg, 9\%) was recovered.3ke: oil; ${ }^{1} \mathrm{H}$ NMR (300 MHz, $\left.\mathrm{CDCl}_{3}\right): \delta 7.59$ (d, $\left.J=7.8 \mathrm{~Hz}, 1 \mathrm{H}\right)$, $7.33(\mathrm{~d}, J=7.8 \mathrm{~Hz}, 1 \mathrm{H}), 7.26(\mathrm{t}, J=7.8 \mathrm{~Hz}, 1 \mathrm{H}), 7.13(\mathrm{t}, J=7.8 \mathrm{~Hz}, 1 \mathrm{H}), 6.92(\mathrm{~s}, 1 \mathrm{H})$, $6.26(\mathrm{~s}, 1 \mathrm{H}), 5.58(\mathrm{~s}, 1 \mathrm{H}), 4.40(\mathrm{q}, J=6.9 \mathrm{~Hz}, 1 \mathrm{H}), 3.80(\mathrm{~s}, 3 \mathrm{H}), 3.77(\mathrm{~s}, 3 \mathrm{H}), 1.59(\mathrm{~d}, J=$ $6.9 \mathrm{~Hz}, 3 \mathrm{H}) ;{ }^{13} \mathrm{C} \mathrm{NMR}\left(75 \mathrm{MHz}, \mathrm{CDCl}_{3}\right): \delta 167.8,144.9,137.2,126.8,126.2,123.8,121.4$, 119.5, 118.6, 117.6, 109.1, 51.7, 32.6, 32.0, 20.3; IR(neat): 1721, 1625, 1465, $1147 \mathrm{~cm}^{-1}$; MS 
$m / z(\%) 243\left(\mathrm{M}^{+}, 100\right)$; HRMS m/z (ESI) calcd for $\mathrm{C}_{15} \mathrm{H}_{17} \mathrm{NO}_{2} \mathrm{Na}^{+}\left(\mathrm{M}^{+}+\mathrm{Na}\right) 266.1151$; Found: 266.1154

(11) 3-(2'-methoxycarbonyl-1'-hexen-3'-yl)-1-methylindole (3kf).<smiles>C=C(C(C)=O)C(c1ccccc1)c1cn(C)c2ccccc12</smiles>

The reaction of $1 \mathbf{k}(66 \mathrm{mg}, 0.5 \mathrm{mmol})$ with $2 \mathrm{f}(323 \mathrm{mg}, 1.5 \mathrm{mmol})$ in the presence of $\mathrm{Pd}(\mathrm{acac})_{2}(14 \mathrm{mg}, 10 \mathrm{~mol} \%)$ and $\mathrm{PPh}_{3}(27 \mathrm{mg}, 20 \mathrm{~mol} \%)$ in $2 \mathrm{~mL}$ of HOAc for $12 \mathrm{~h}$ afforded $61 \mathrm{mg}(45 \%)$ of 3kf: oil; ${ }^{1} \mathrm{H}$ NMR (300 MHz, $\left.\mathrm{CDCl}_{3}\right): \delta 7.59$ (d, $\left.J=7.8 \mathrm{~Hz}, 1 \mathrm{H}\right), 7.28(\mathrm{~d}, J$ $=7.8 \mathrm{~Hz}, 1 \mathrm{H}), 7.21(\mathrm{t}, J=7.8 \mathrm{~Hz}, 1 \mathrm{H}), 7.08(\mathrm{t}, J=7.8 \mathrm{~Hz}, 1 \mathrm{H}), 6.89(\mathrm{~s}, 1 \mathrm{H}), 6.22(\mathrm{~s}, 1 \mathrm{H})$, $5.62(\mathrm{~s}, 1 \mathrm{H}), 4.20(\mathrm{t}, J=7.5 \mathrm{~Hz}, 1 \mathrm{H}), 3.75(\mathrm{~s}, 3 \mathrm{H}), 3.73(\mathrm{~s}, 3 \mathrm{H}), 1.95-1.87(\mathrm{~m}, 2 \mathrm{H})$, 1.45-1.32 (m, $2 \mathrm{H}), 0.94(\mathrm{t}, J=7.2 \mathrm{~Hz}, 3 \mathrm{H}) ;{ }^{13} \mathrm{C} \mathrm{NMR}\left(75 \mathrm{MHz}, \mathrm{CDCl}_{3}\right): \delta$ 167.9, 144.1, 137.1, 127.3, 126.6, 123.7, 121.4, 119.5, 118.6, 116.3, 109.1, 51.8, 37.4, 36.8, 32.7, 21.2, 14.1; IR(neat): 1719, 1626, 1469, $1149 \mathrm{~cm}^{-1}$; MS m/z (\%) 228 (100), $271\left(\mathrm{M}^{+}, 41.59\right) ;$ HRMS m/z (EI) calcd for $\mathrm{C}_{17} \mathrm{H}_{21} \mathrm{NO}_{2}{ }^{+}\left(\mathrm{M}^{+}\right)$271.1572; Found: 271.1585. 

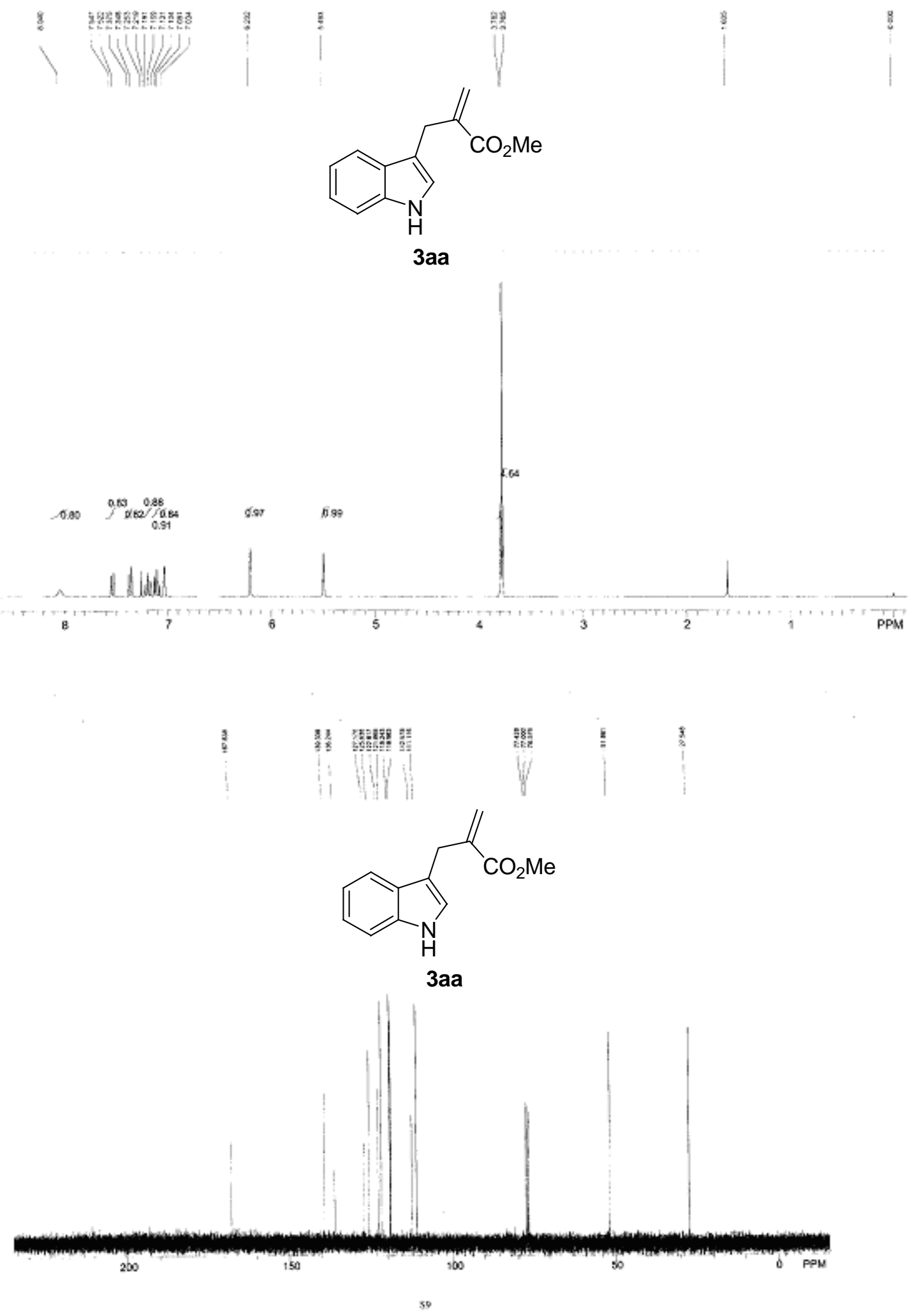

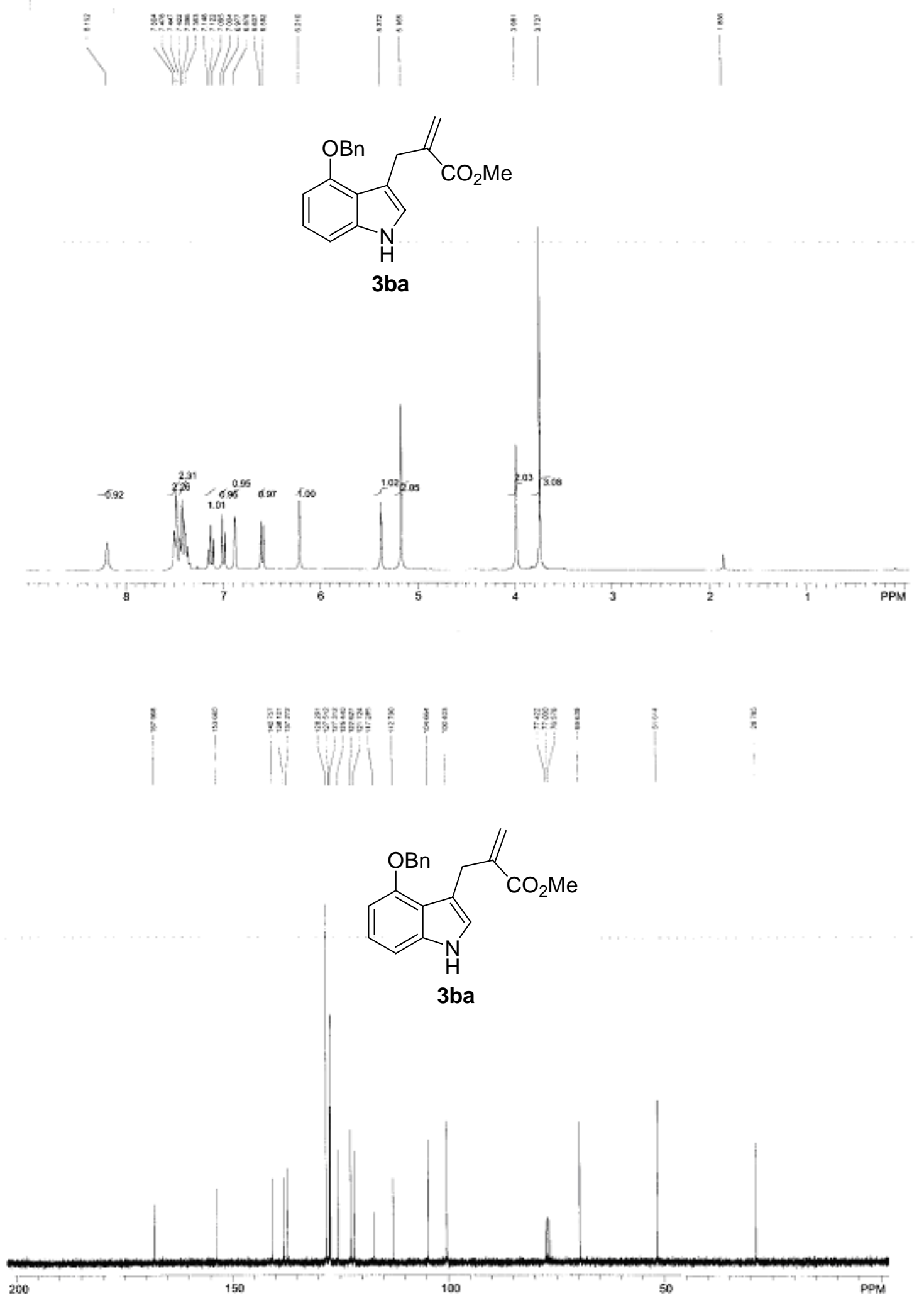
$y=c-11-83.0$

Archive directory: /export/home/masm/vnnrsys/date
Sanple directory:

Pulse Sequence: s2pul

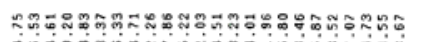

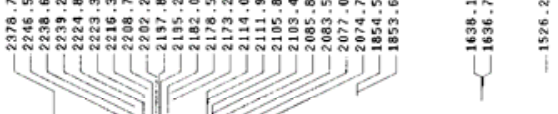
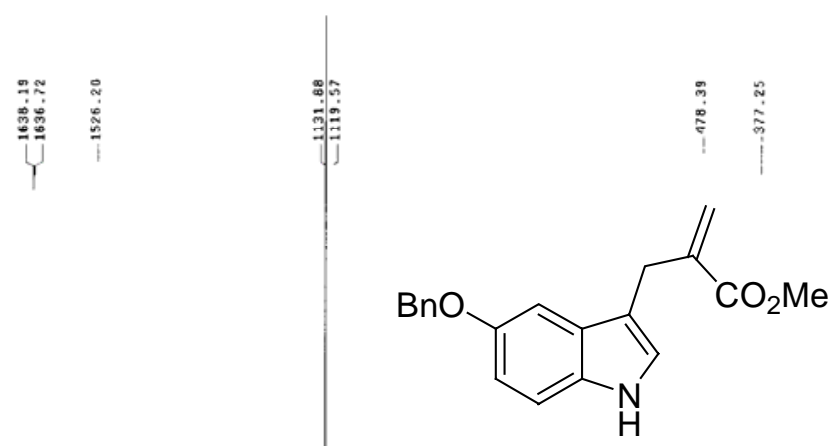

$3 c a$
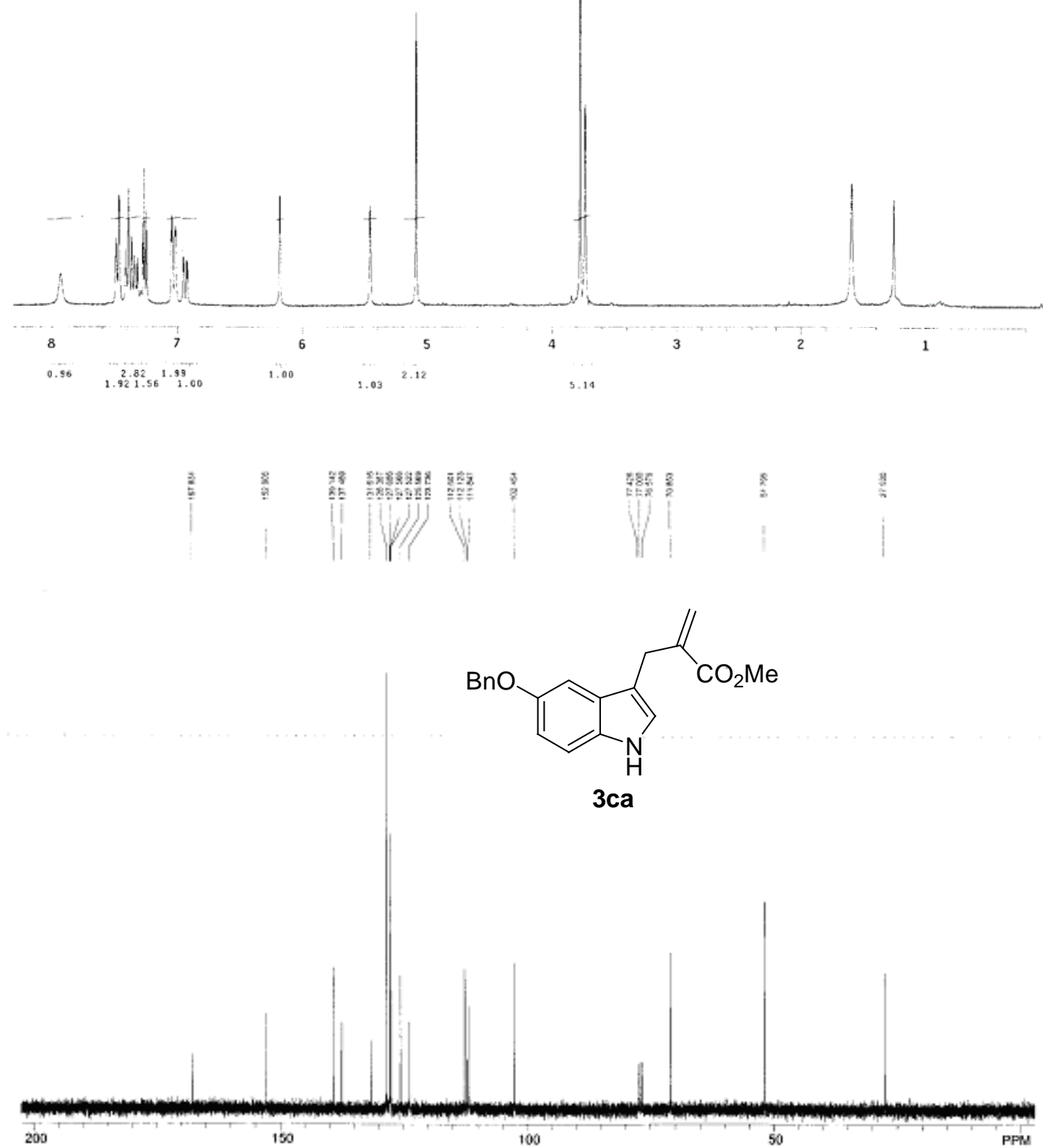

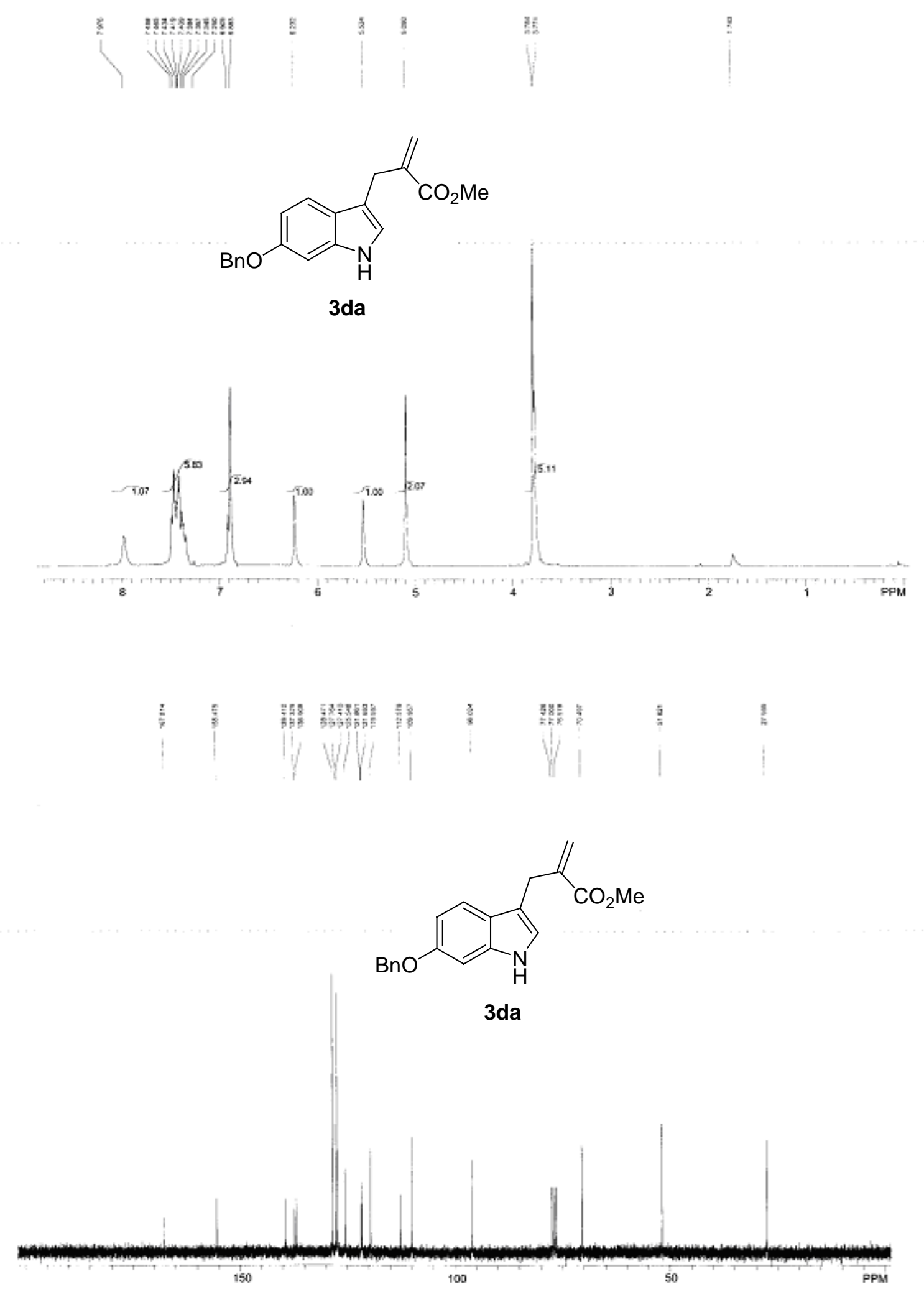

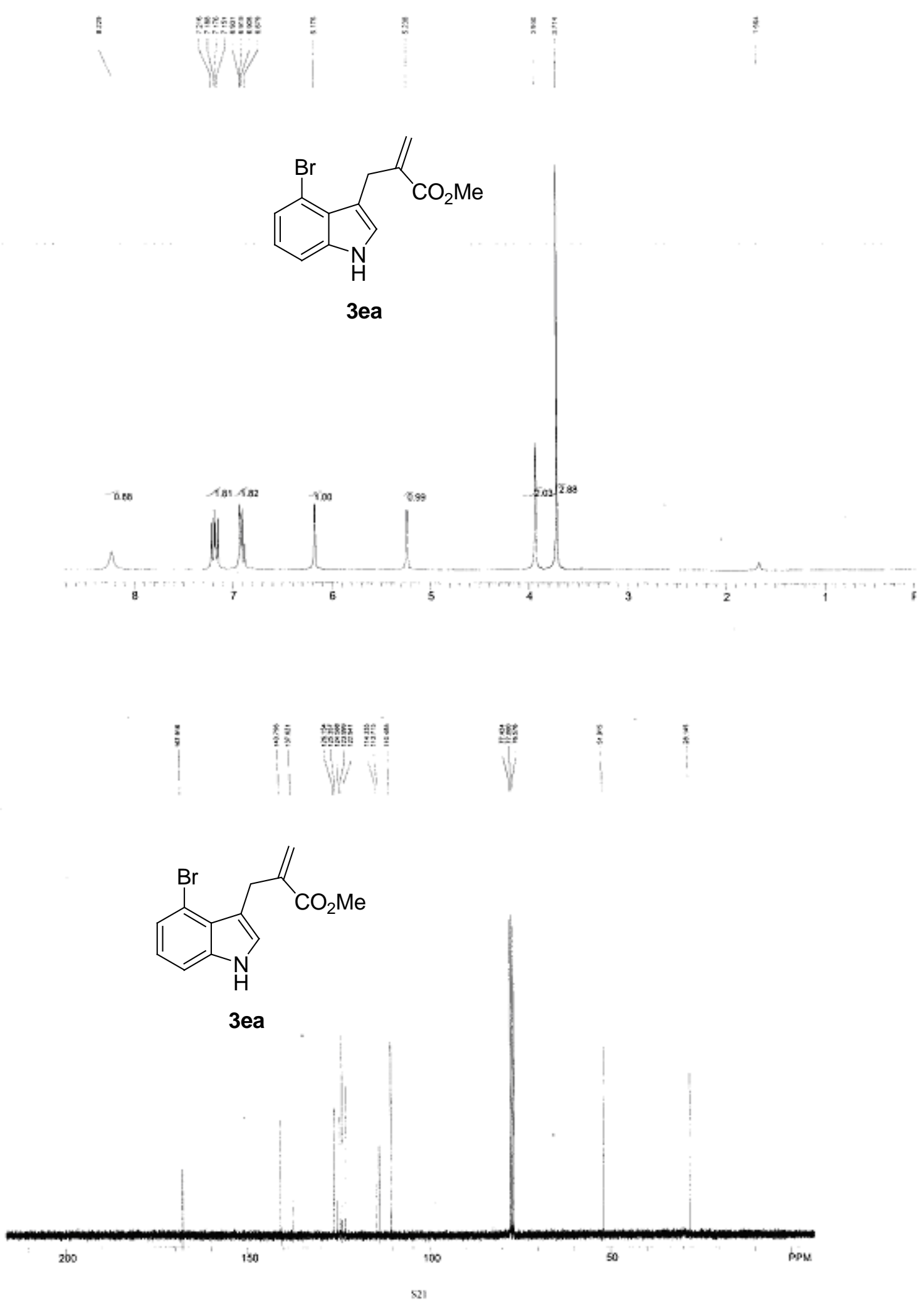


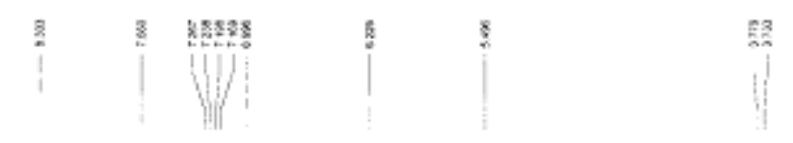
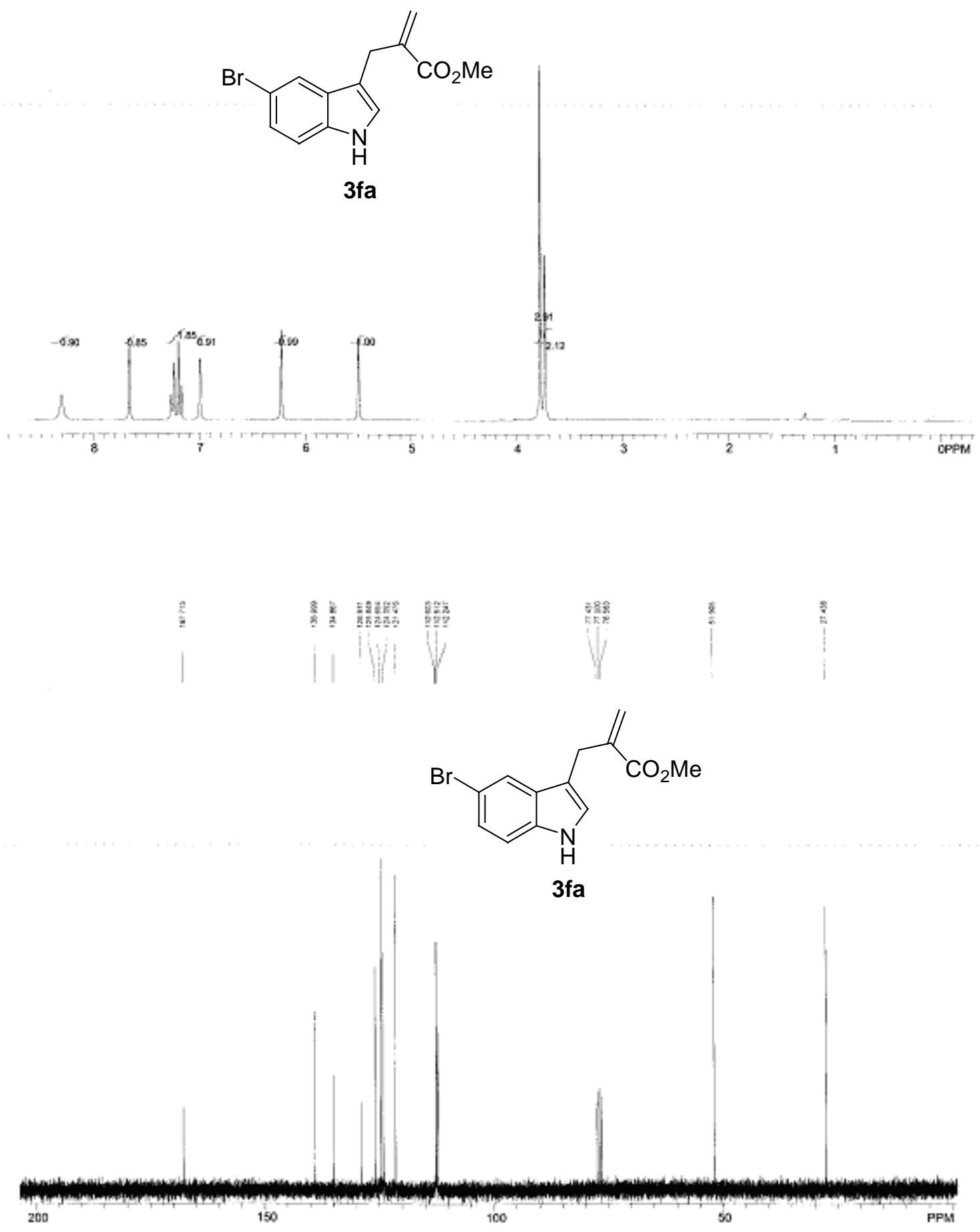

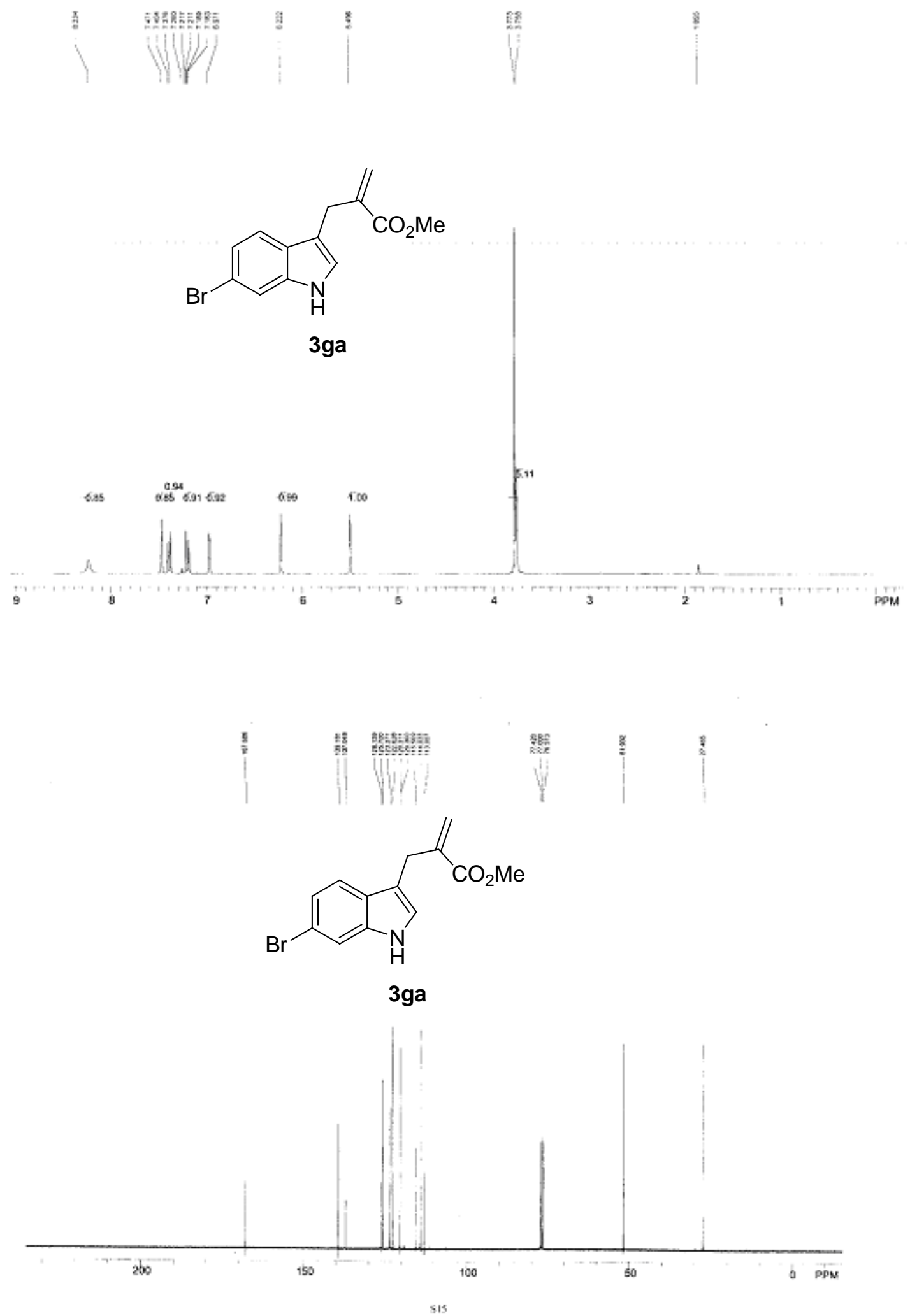

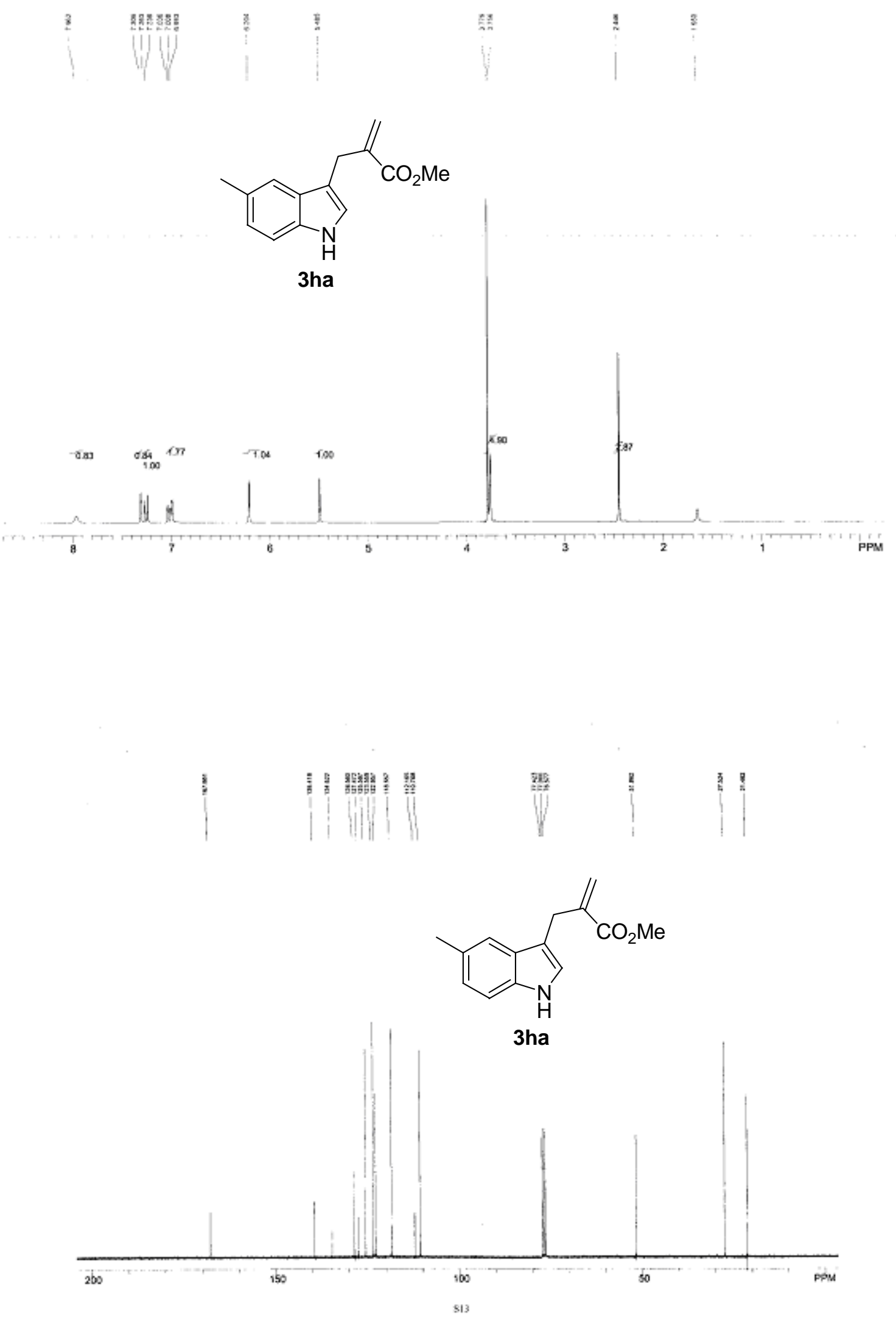


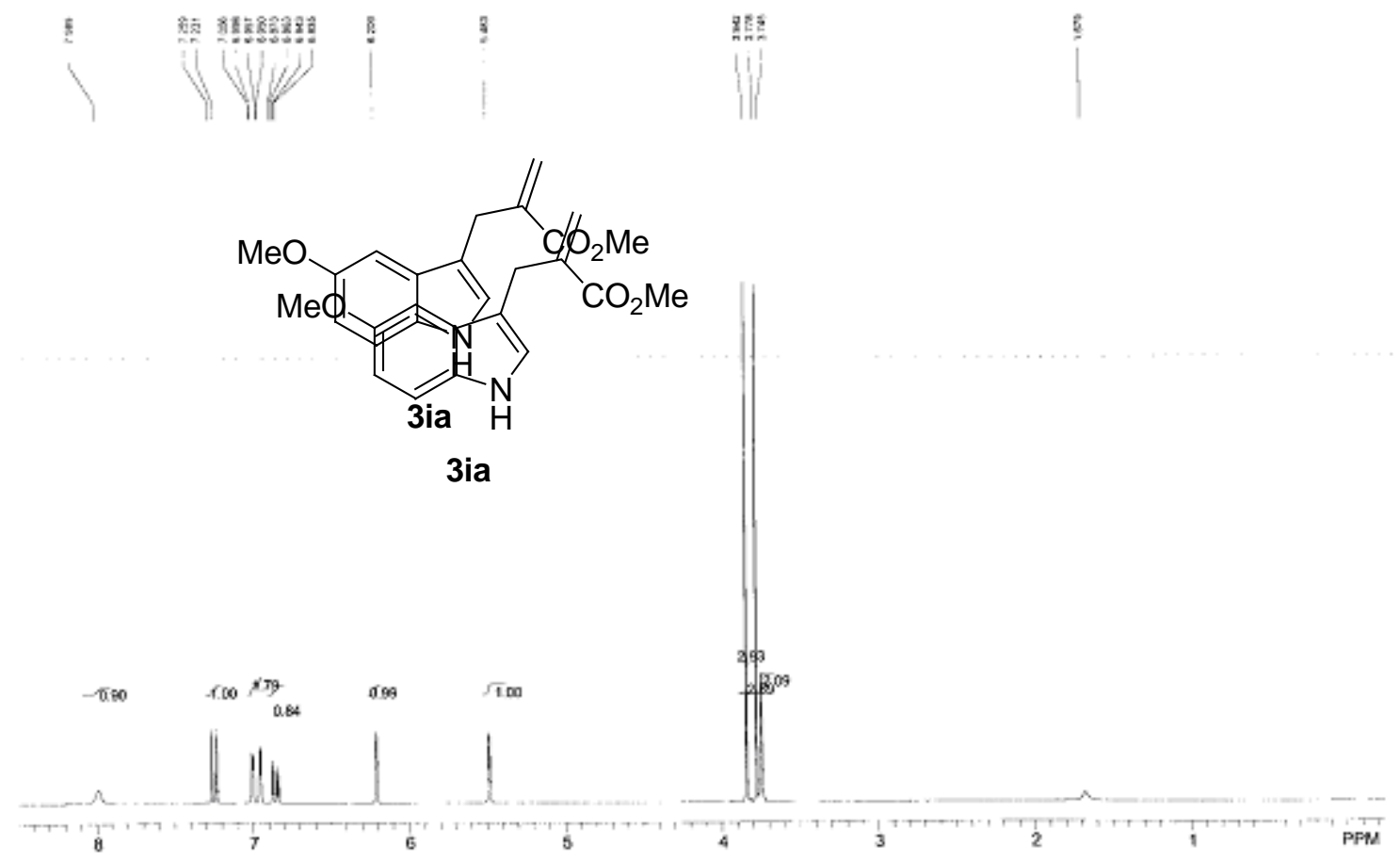

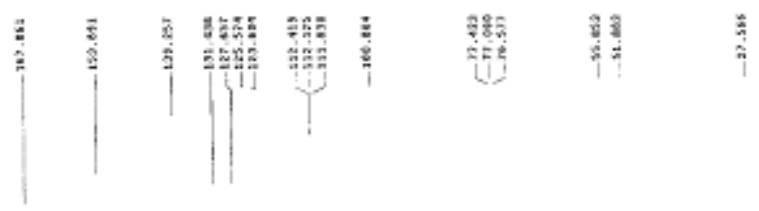

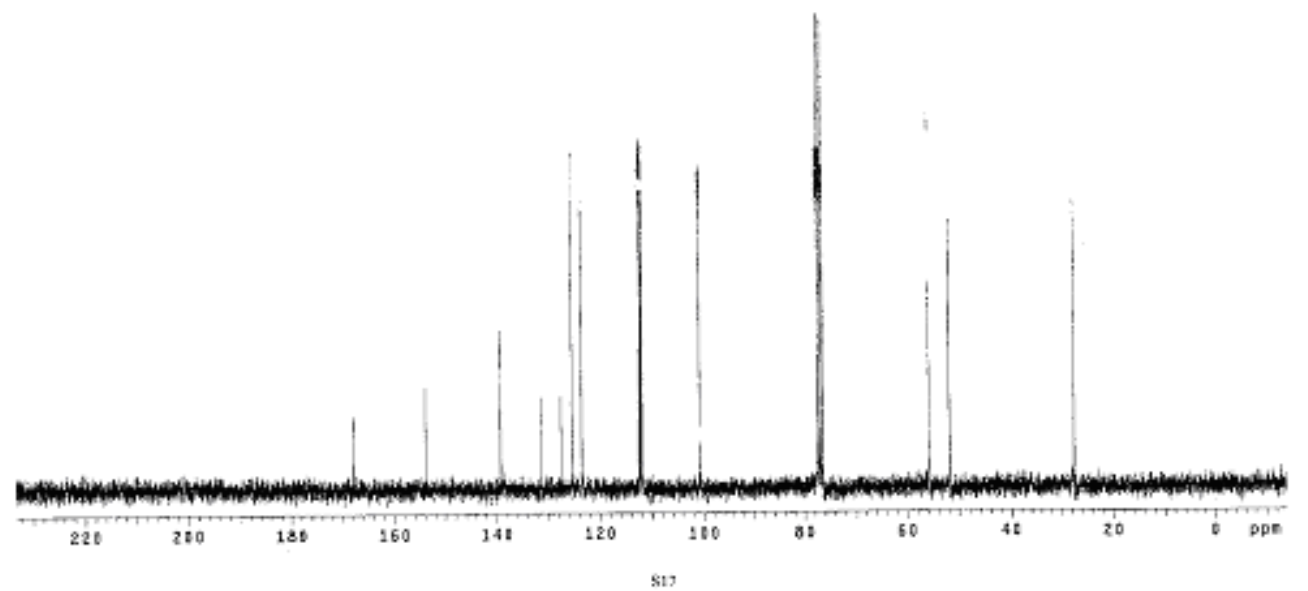




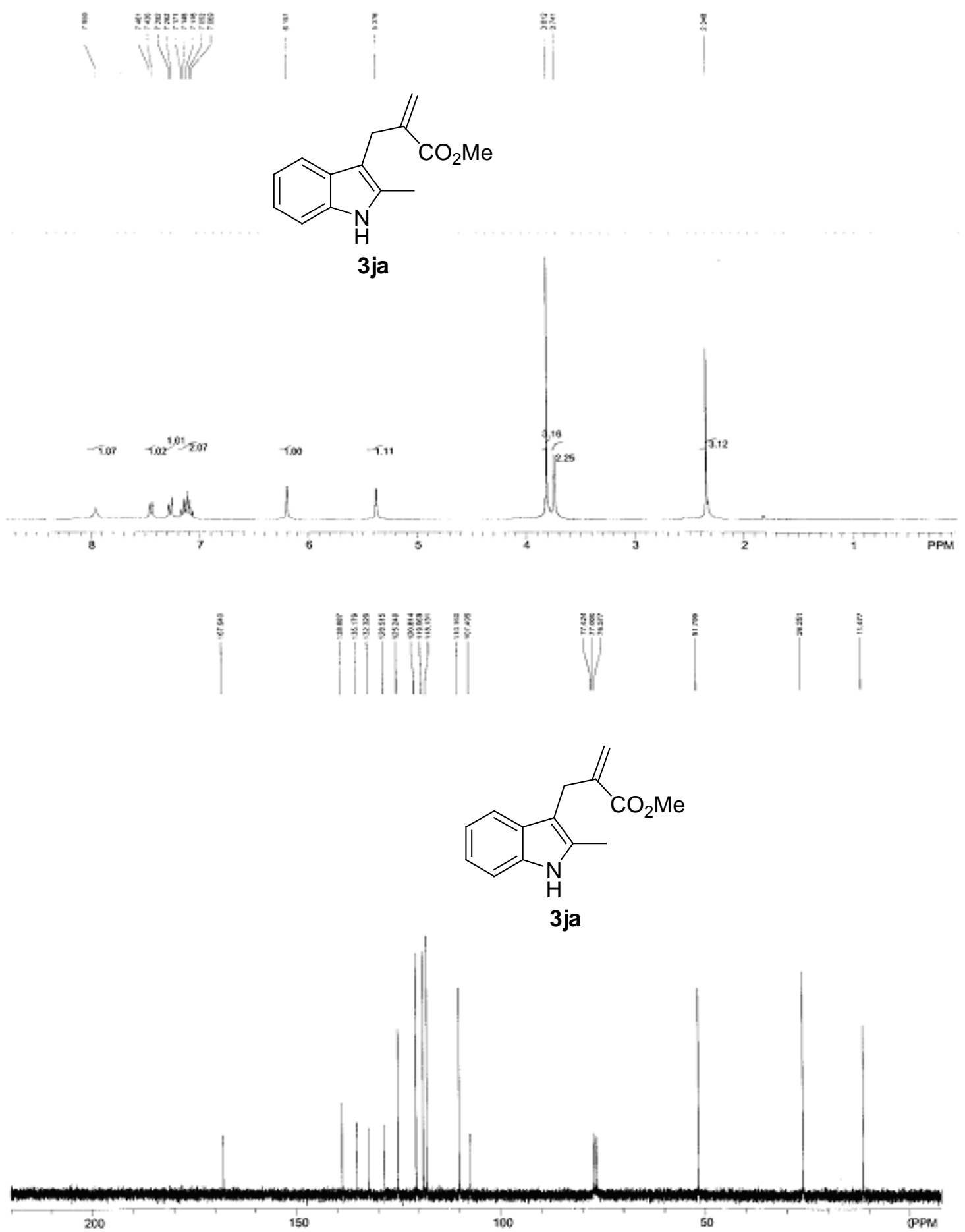



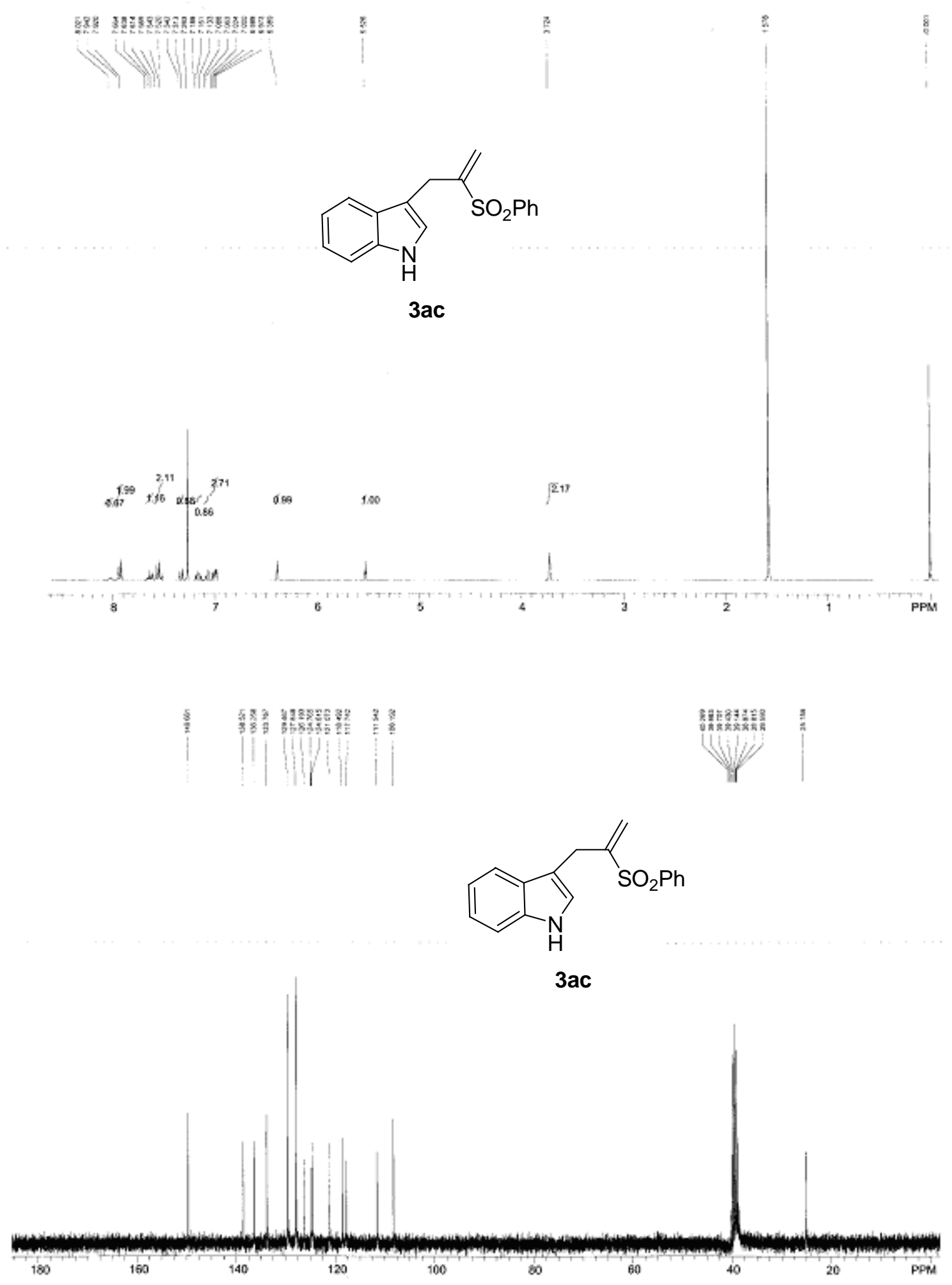

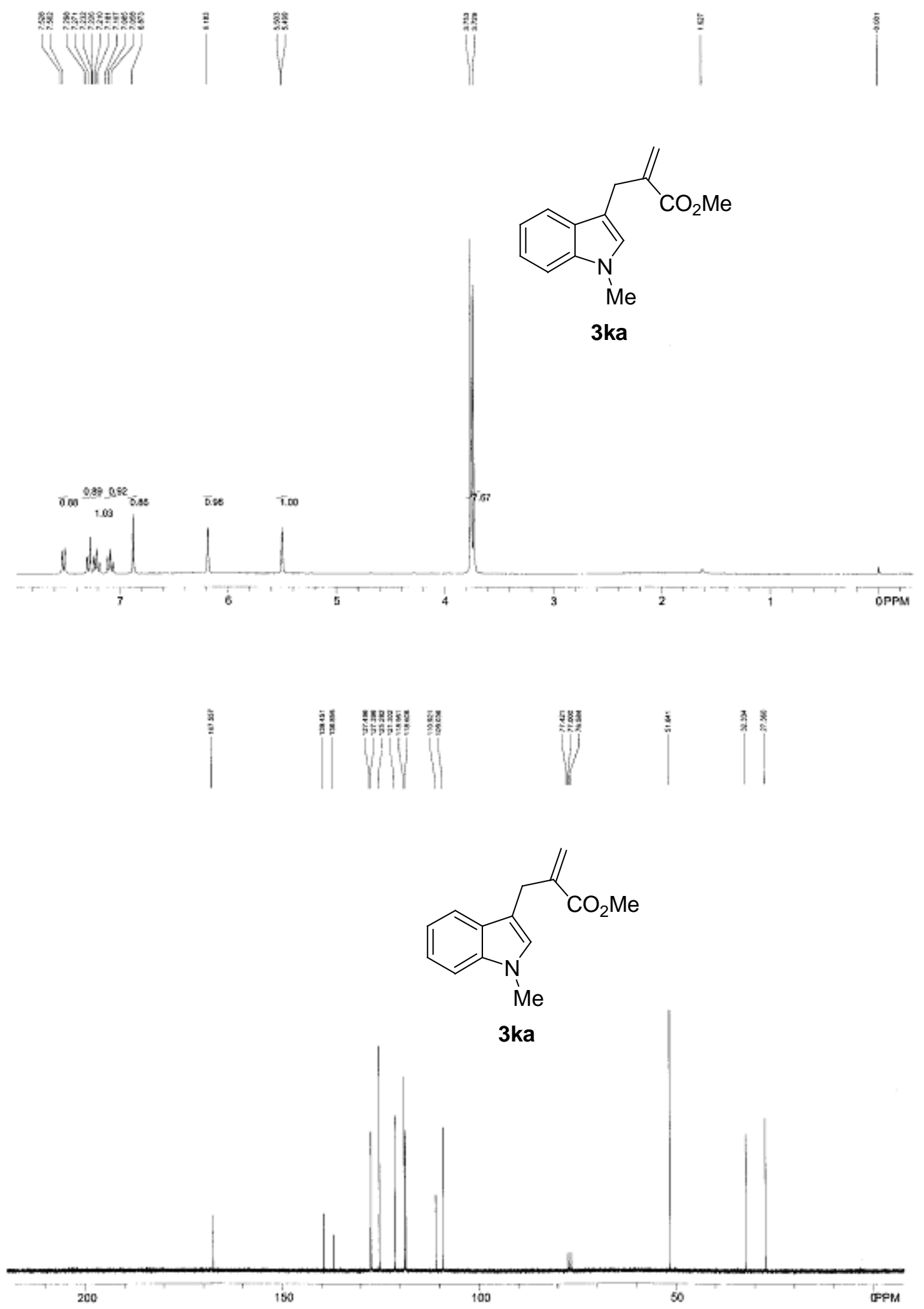

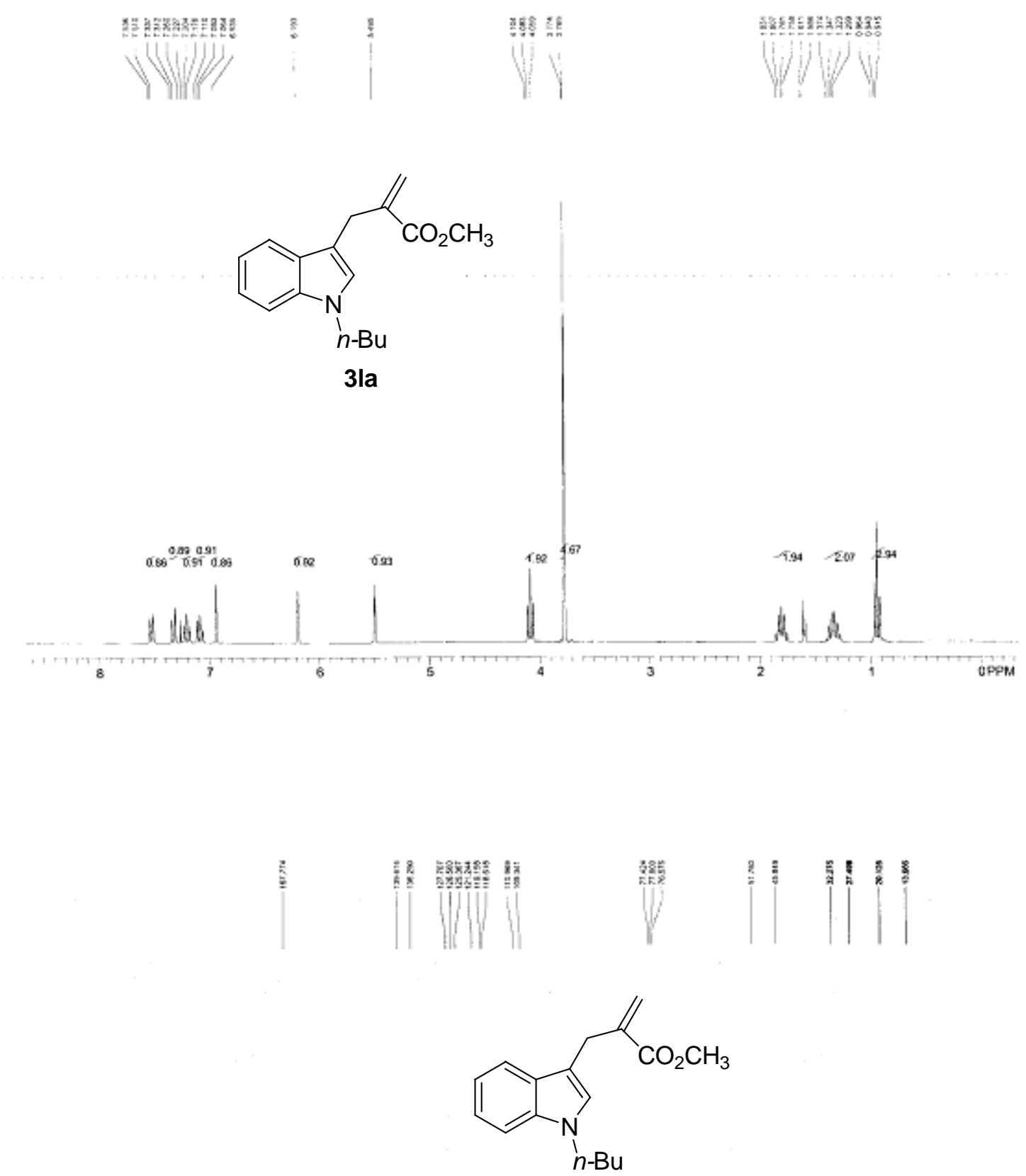

3la

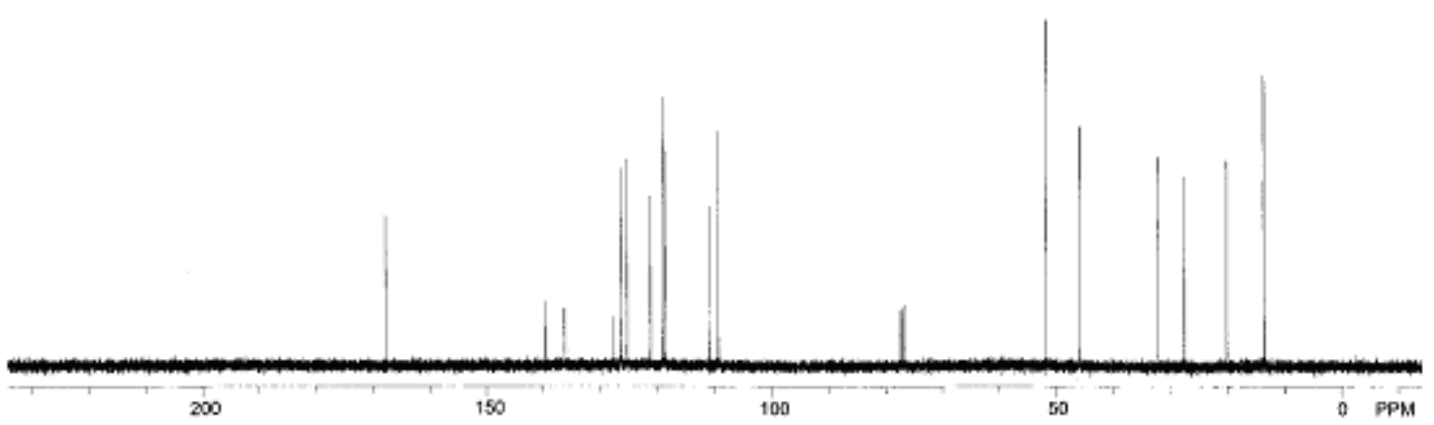



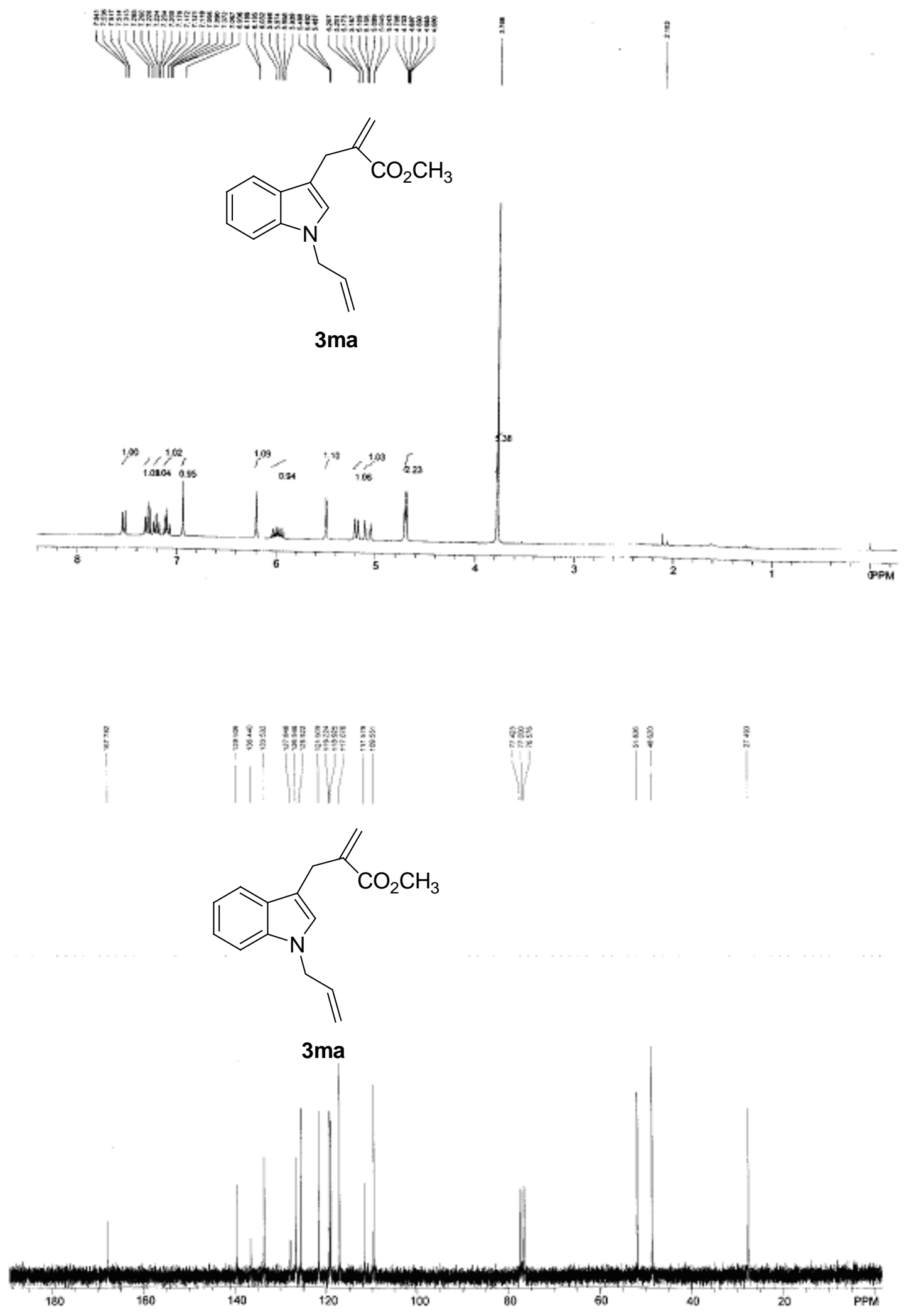

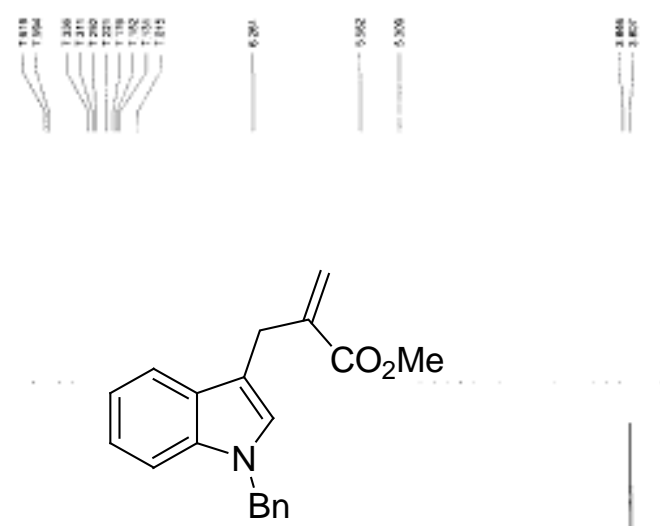

3na
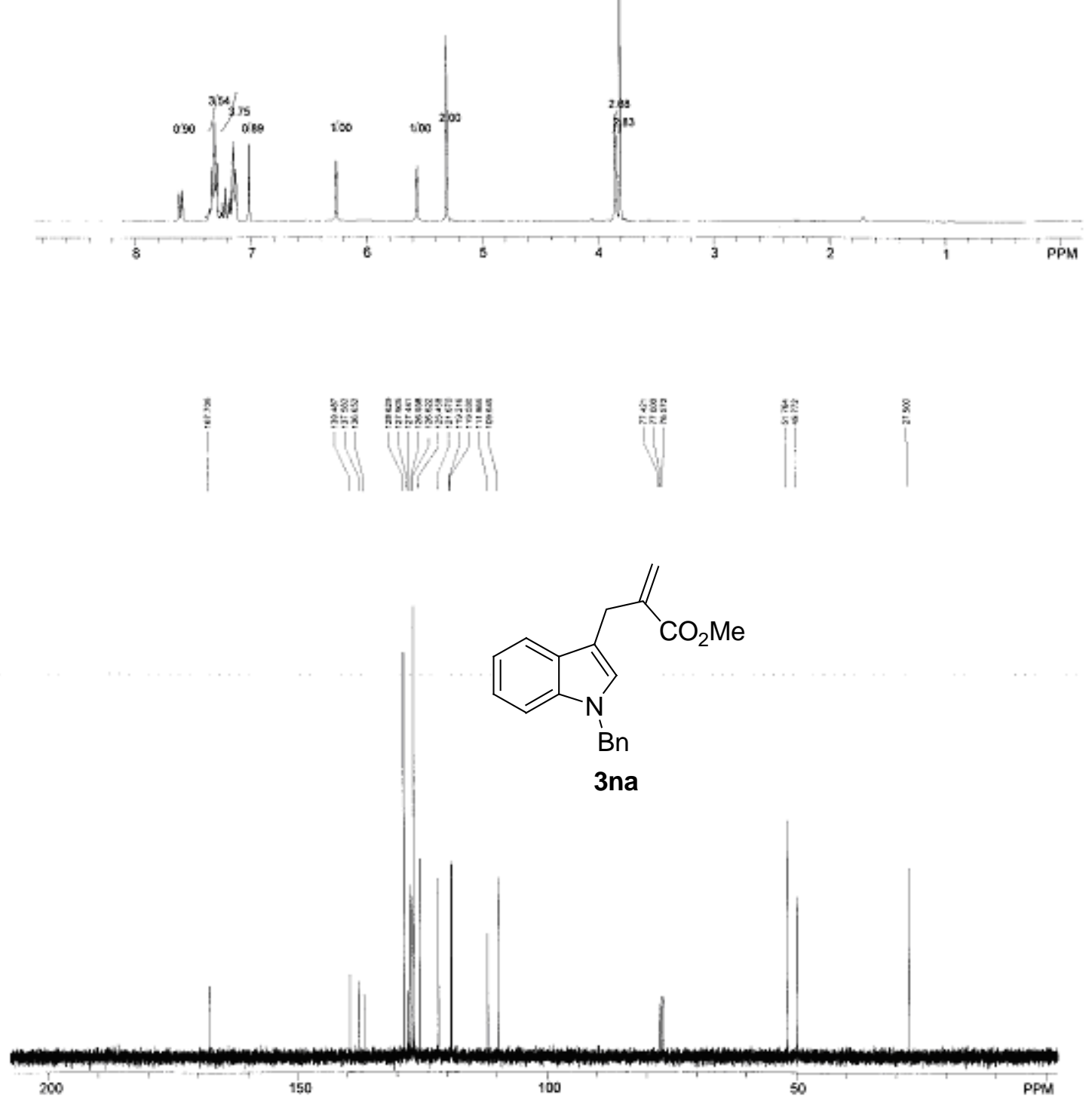


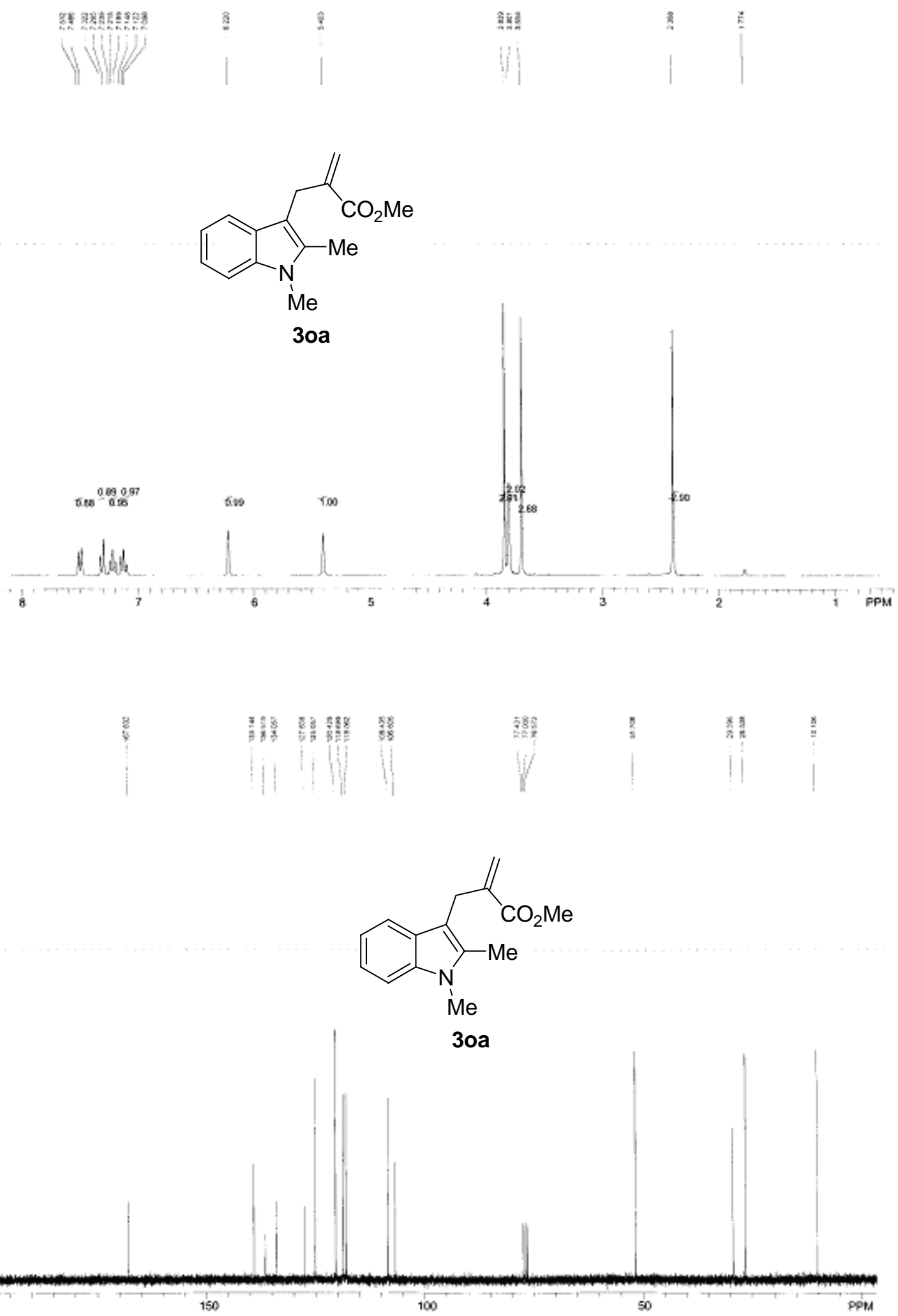



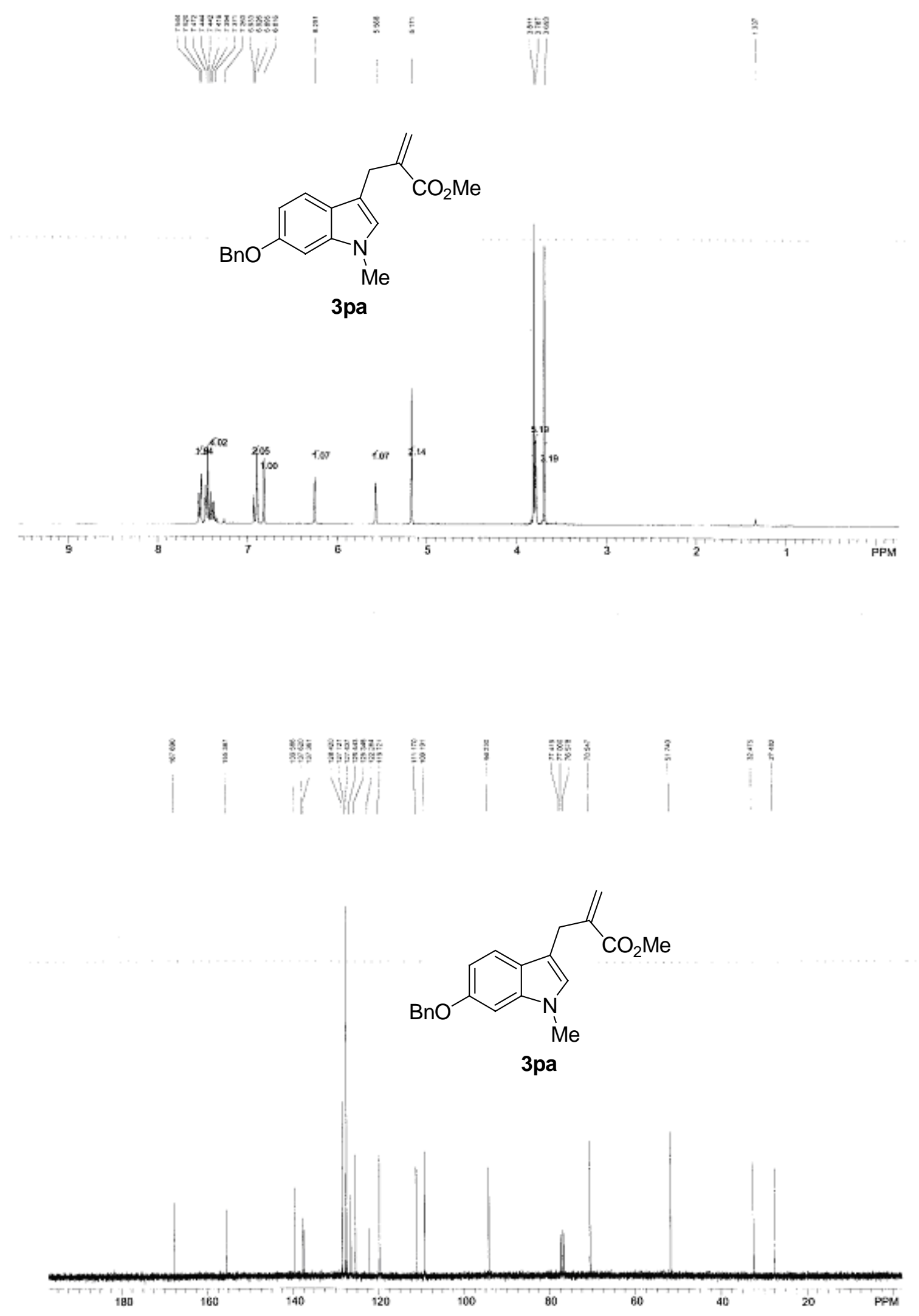
<smiles>C=C(Cc1cn(C)c2ccccc12)C(C)=O</smiles>

$3 k b$

$$
0.500_{0.58}^{0.998}{ }_{0.97}^{0.98}
$$

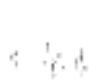

7
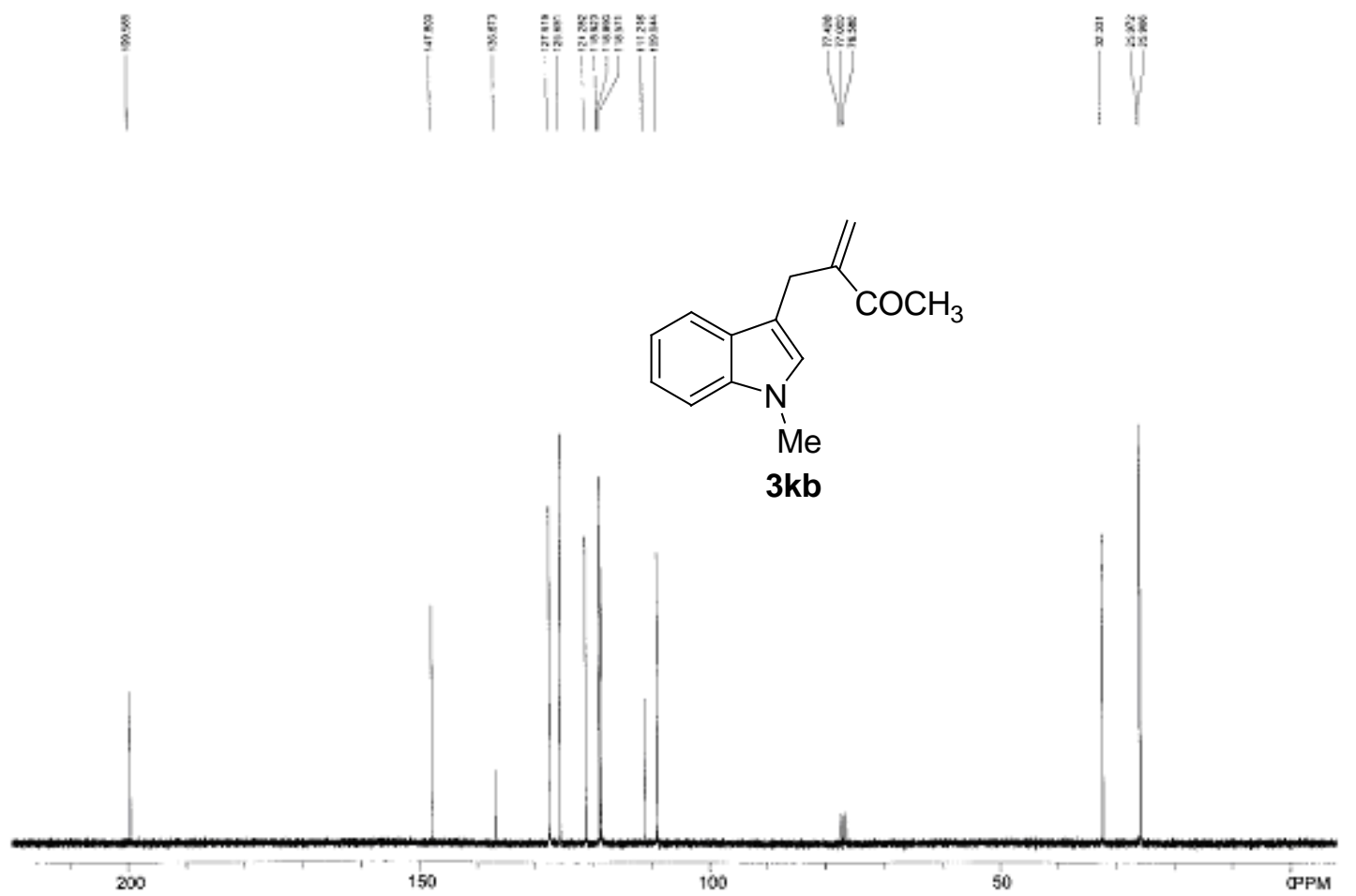

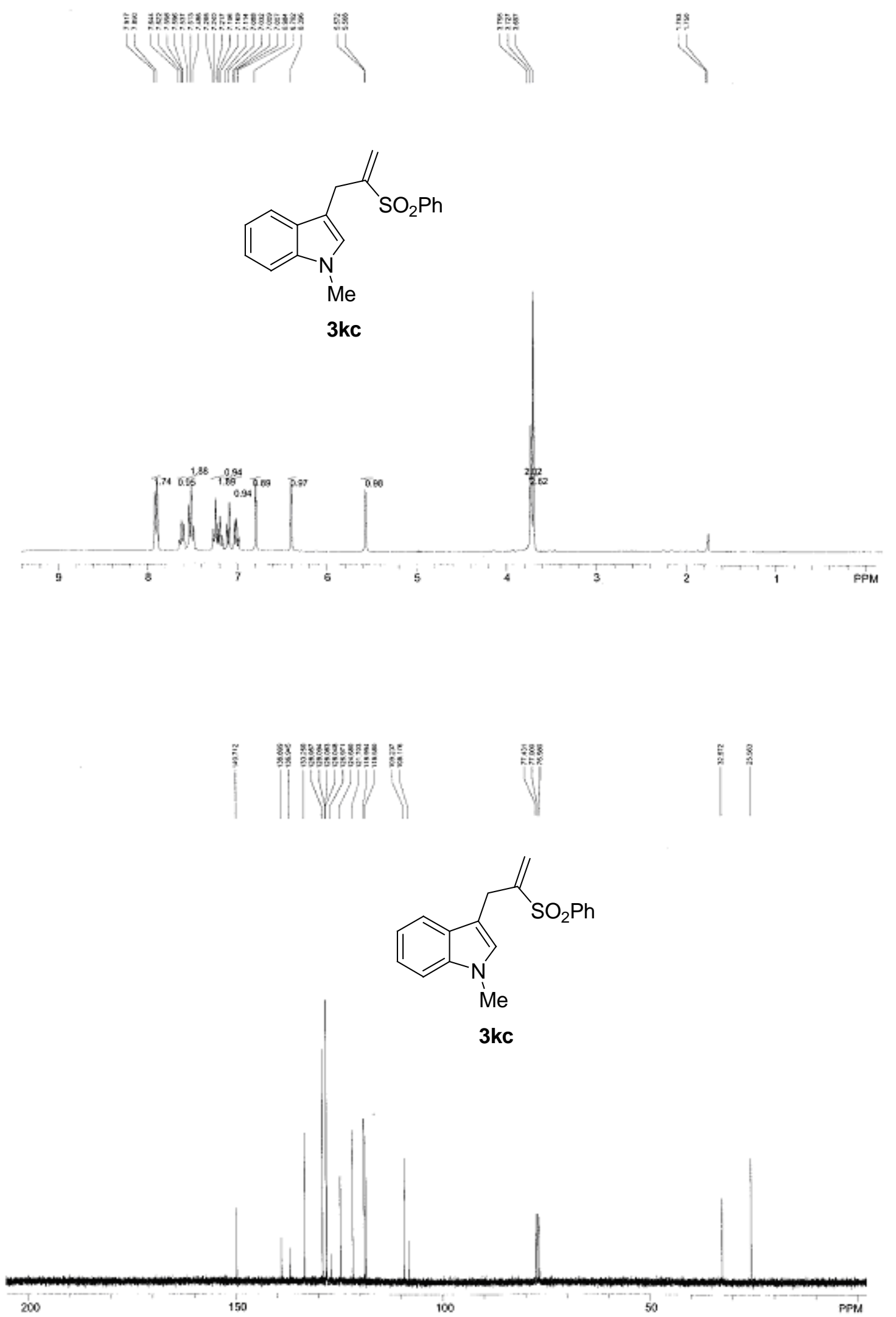


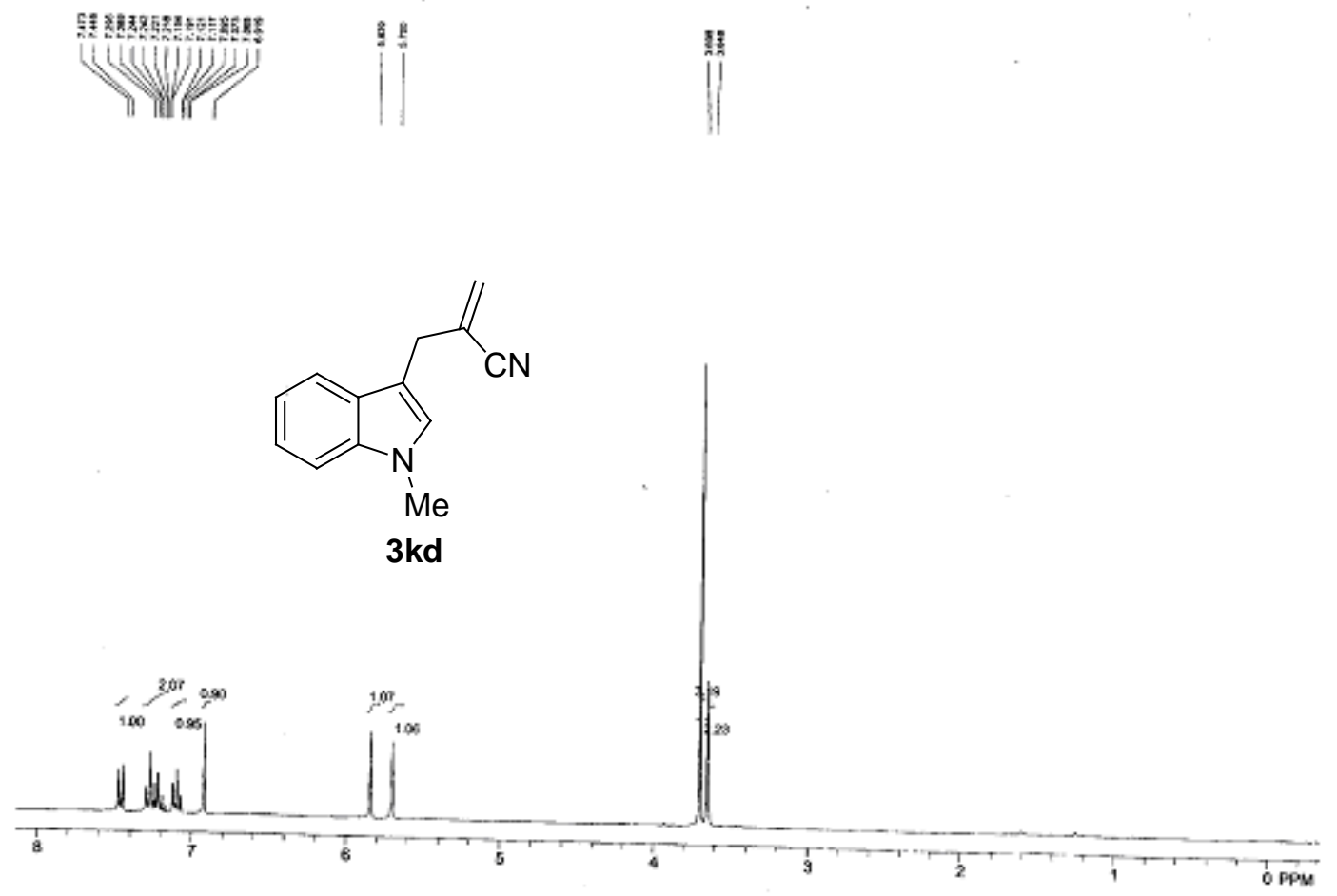

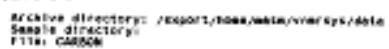

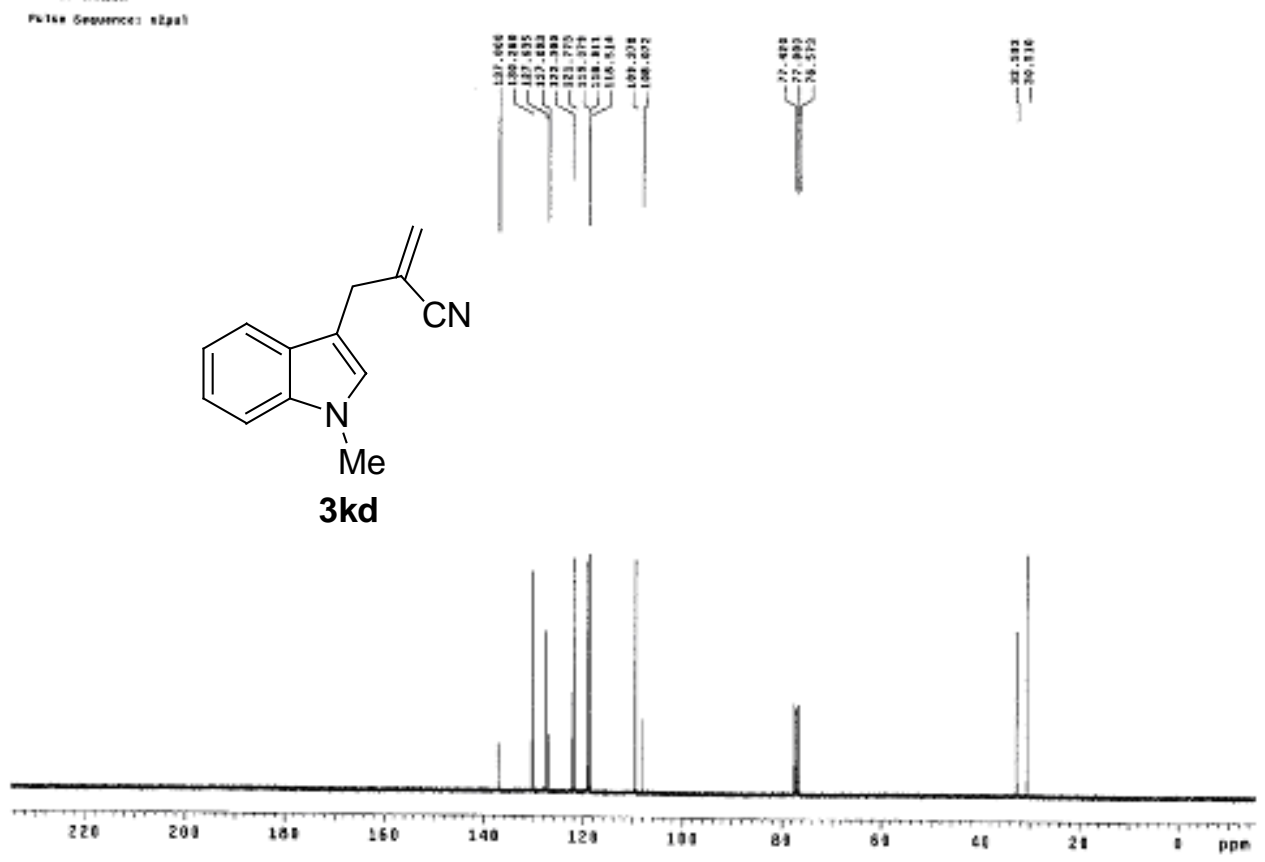




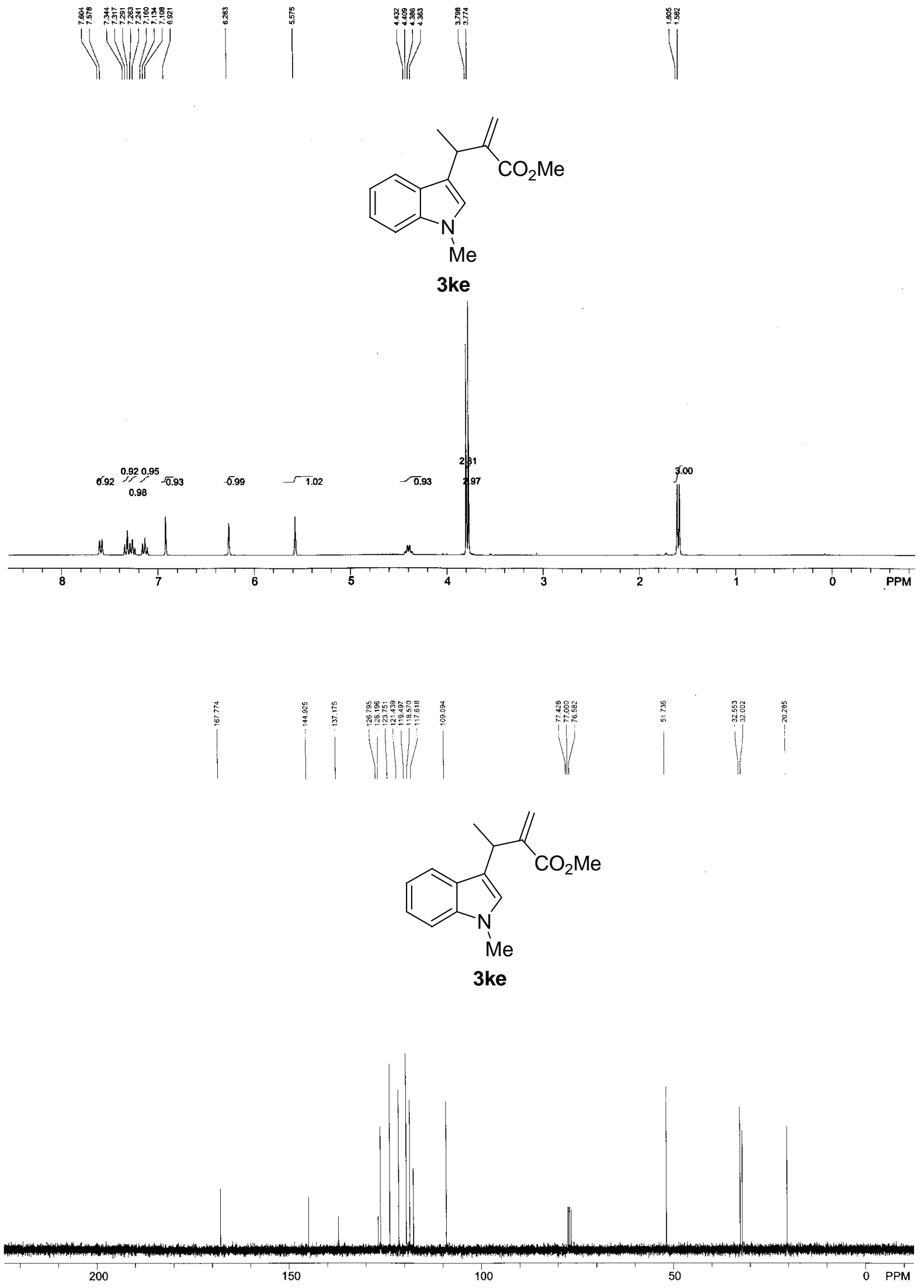



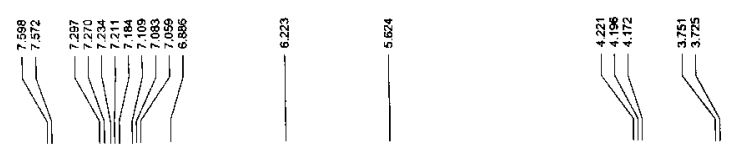

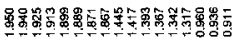

(uij) (il)<smiles>C=C(C(C)=O)C(c1ccccc1)c1cn(C)c2ccccc12</smiles>

3kf
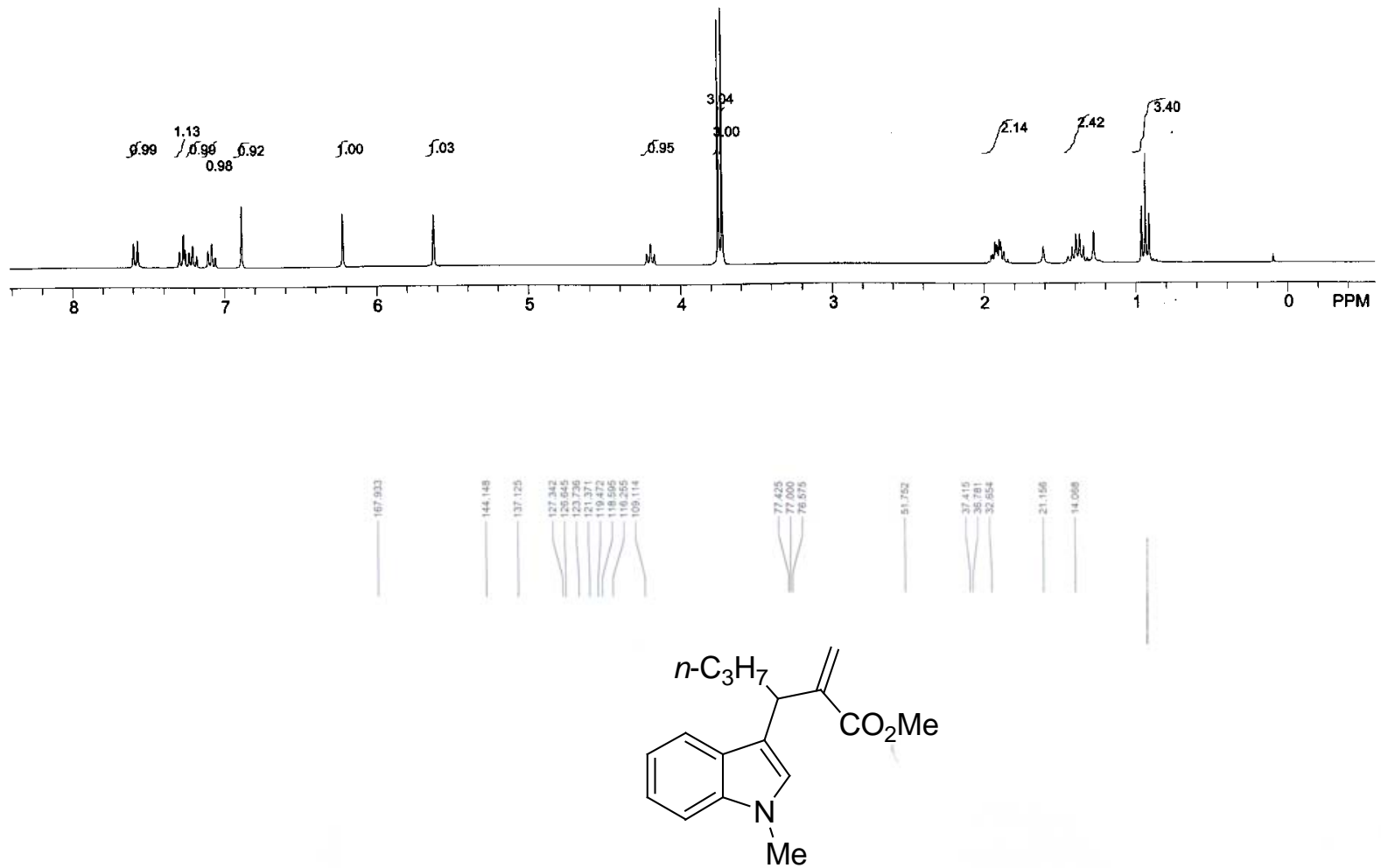

3kf

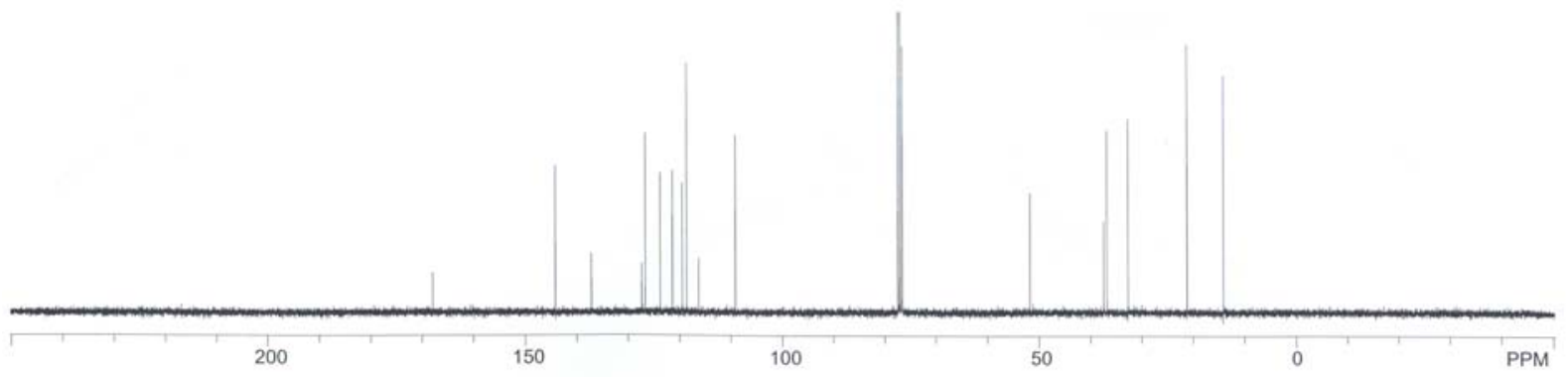

